\title{
General Properties of Option Prices
}

\author{
Yaacov Z. Bergman ${ }^{1}$, Bruce D. Grundy ${ }^{2}$ and Zvi Wiener ${ }^{3}$
}

Forthcoming: The Journal of Finance

First Draft: February 1995

Current Draft: January 1996

1 The School of Business and Center for Rationality, Hebrew University, Jerusalem. msyberg@pluto.mscc.huji.ac.il

2 The Wharton School, University of Pennsylvania. grundy@wharton.upenn.edu

3 The School of Business, Hebrew University, Jerusalem. mswiener@pluto.mscc.huji.ac.il

This paper is a substantially revised version of an earlier work previously circulated as Theory of Rational Option Pricing: II. The authors are grateful for helpful discussions with Kaushik Amin, Giovanni Barone-Adesi, Avi Bick, Peter Carr, Domenico Cuoco, Sanjiv Das, Darrell Duffie, Phil Dybvig, Mark Garman, Steve Grenadier, Sanford Grossman, Jon Ingersoll, Alexander Nabutovsky, Steve Ross and Mark Rubinstein, and for the comments of workshop participants at the Wharton School, Berkeley, Carnegie-Mellon, Chicago, Harvard, Hebrew, Houston and Maryland Universities, Washington University in St. Louis, the NBER Financial Risk Assessment and Management Conference, the Sixth Annual Conference in Financial Economics \& Accounting, the 1995 European Finance Association Meetings, the Sixth Summer Institute on Game Theory at SUNY Stony Brook and the 1996 American Finance Association Meetings. The authors gratefully acknowledge financial support from the H. Krueger Center for Finance at the Hebrew University (Bergman and Wiener), a Batterymarch Fellowship and the Geewax-Terker Program in Financial Instruments (Grundy) and a Rothschild Fellowship (Wiener). 


\title{
General Properties of Option Prices
}

\begin{abstract}
When the underlying price process is a one-dimensional diffusion, as well as in certain restricted stochastic volatility settings, a contingent claim's delta is always bounded by the infimum and supremum of its delta at maturity. Further, if the claim's payoff is convex (concave), then the claim's price is a convex (concave) function of the underlying asset's value. However when volatility is less specialized, or when the underlying price follows a discontinuous or non-Markovian process, then call prices can have properties very different from those of the Black-Scholes model: a call's price can be a decreasing, concave function of the underlying price over some range; increasing with the passage of time; and decreasing in the level of interest rates.
\end{abstract}

Much of the financial options literature derives precise option prices, when the underlying asset price process is completely specified. Since it is empirically difficult to ascertain what the true underlying process is, another part of that literature is concerned with deriving general properties of option prices, when the underlying price process is not fully specified, but instead is assumed to belong to some general class of stochastic processes (Merton (1973), Cox and Ross (1976), Jagannathan (1984)). In particular, in the absence of arbitrage opportunities and assuming that the risk-neutralized stock price follows a proportional stochastic process (a risk-neutral return distribution independent of price), Merton (1973) and Jagannathan (1984) show that a call option's price is an increasing, convex function of the stock price. Cox and Ross (1976) generalize this result and show that, under the same proportionality assumption of the stock price process, the price function of any European contingent claim, not just a call option, inherits qualitative properties of the claim's contractual payoff function. Merton (1973) notes that although convexity is usually assumed to be a property that holds for calls, proportionality of the stock price process is not a necessary condition for that. He thus implicitly conjectures that quite general but different conditions exist, that generate the same results. 
A class of stochastic processes, that traditionally have played a prominent role in modeling the dynamics of underlying prices, are the diffusions. It is then important to verify whether the increasing and convex call price result of Merton (1973) and Jagannathan (1984), and the Cox and Ross (1976) generalization thereof, is valid for that class as well. Indeed, when the underlying process belongs to a quite general class of diffusions, we obtain the above results and more. Specifically, we establish that whenever the underlying asset follows a diffusion whose volatility depends only on time and the concurrent stock price, then a call price is always increasing and convex in the stock price, decreasing with the passage of time, and increasing in the level of interest rates. However, when volatility is stochastic, or the stock price process is not a diffusion, but is instead either discontinuous or non-Markovian, we show that a call price can be a decreasing, concave function of the stock price over some range, that a call can be a 'bloating' - not a 'wasting' asset, and that an increase in interest rates can lead to a decline in a call price.

Our analysis is not limited to call options. We establish properties of any European-style contingent claims given deterministic interest rates and various specifications of the underlying asset price process. Whenever the underlying asset follows a one-dimensional diffusion, properties of the stock and bond positions in a contingent claim's replicating portfolio are shown to be inherited from those at maturity. Specifically, the positions are bounded by the infimum and supremum of those positions at maturity. If the position in stock at maturity is increasing (decreasing) in the underlying maturity-date price, then that position - the claim's 'delta'-is increasing (decreasing) in the concurrent underlying price. Equivalently, if the claim's contractual payoff function is convex (concave) in the underlying price at expiration, then the claim's price is convex (concave) in the concurrent underlying price. Analogous results are established for certain restricted multidimensional diffusion (stochastic volatility) settings.

Armed with these results about general properties of contingent claim prices, we are able to extend comparative static results familiar in a Black-Scholes setting to that of a general onedimensional diffusion. We consider the effects of changes in interest rates, in dividend rates, and in volatility on the prices of call options. In doing so we derive three additional results. Although 
an upward shift in the entire term structure will always increase call prices, a twist in the termstructure that decreases the present value of a call's exercise price can decrease its value. We establish a new bound on the relative values of options on otherwise equivalent dividend-paying and non-dividend-paying assets in terms of the fraction of the dividend-paying asset's price that is due to dividends expected beyond the call's maturity. Our analysis of differing volatility functions establishes that when the underlying asset's volatility is bounded above (below), then, whatever the functional form of the relation between volatility, time, and the contemporaneous stock price, the call price is bounded above (below) by its Black-Scholes value calculated at the bounding volatility level. One can then place bounds on the stock position necessary to hedge a given option position using only knowledge of the bounds on the underlying asset's volatility.

The plan of the paper is as follows. Section I establishes properties of general contingent claims that are satisfied in all one-dimensional diffusion settings, and in certain restricted forms of a multi-dimensional diffusion setting. Section II applies those properties to the pricing of call options. Section III contains the comparative statics analyses. Section IV establishes that call options need not possess any of their familiar properties, if either volatility is stochastic and not restricted in the manner considered in Section I, or the underlying process is not a diffusion, but is instead either discontinuous or non-Markovian. Section V summarizes our results.

\section{A Diffusion Process for the Underlying Asset}

Consider a European contingent claim maturing at time $T$. The time $t$ price of the traded underlying asset is denoted by $s_{t}$. We assume that the stochastic process describing changes in $s_{t}$ admits no arbitrage opportunities and is either a one- or multi-dimensional diffusion, as defined next.

Definition 1. The underlying asset will be said to follow a one-dimensional diffusion when

$$
d s_{t}=\alpha(\cdot) d t+\sigma\left(s_{t}, t\right) s_{t} d B_{t}
$$

The instantaneous volatility, $\sigma(\cdot)$, is a function of $s_{t}$ and $t$ only, while the drift parameter, $\alpha(\cdot)$, is not necessarily so restricted. $B_{t}$ denotes a standard Brownian motion. 
We follow the finance literature and refer to $\sigma(\cdot)$, rather than $\sigma(\cdot) s$, as the volatility. Following Karlin and Taylor (1981, p. 159) we refer to the product $\sigma(\cdot)$ s as the diffusion parameter. The functions $\alpha(\cdot)$ and $\sigma(\cdot)$ are assumed to satisfy whatever regularity conditions are necessary for (1) to be a well-defined stochastic differential equation. ${ }^{1}$ We refer to the special case when volatility is deterministic, $\sigma(t)$, as a Black-Scholes setting.

Definition 2. The underlying asset will be said to follow a two-dimensional diffusion when

$$
\begin{aligned}
& d s_{t}=\alpha(\cdot) d t+\sigma\left(s_{t}, y_{t}, t\right) s_{t} d B_{t}^{1}, \\
& d y_{t}=\beta\left(s_{t}, y_{t}, t\right) d t+\theta\left(s_{t}, y_{t}, t\right) d B_{t}^{2}
\end{aligned}
$$

and $d B_{t}^{1} d B_{t}^{2}=\rho(s, y, t) d t$. [Superscripts on $d B_{t}$ are indices - not powers.]

The underlying asset price dynamics in (2) are usually referred to in the finance literature as a setting with stochastic volatility, although, clearly, the volatility in the one-dimensional case need not be deterministic. Our results are valid for a vector $y_{t}$ as well, but for ease of exposition, only a one-dimensional diffusion $y_{t}$ is considered, so that changes in $s_{t}$ are driven by a two-dimensional diffusion.

We use the terms one-dimensional case and two-dimensional case to mean, respectively, definitions 1 and 2. Unless otherwise noted, it is assumed that underlying assets pay no dividends over the life of the contingent claim. Interest rates are assumed to be deterministic; functions of time, at most. ${ }^{2}$

Let $v(s, t)$ denote the time $t$ value of a contingent claim in the one-dimensional case. (When, in particular, a call option is considered, $c$ instead of $v$ will be used to denote its price.) Numerical subindices denote partial derivatives. Thus, for example, $v_{11}(s, t)$ is the second partial with respect to the first argument; the stock price. We consider only limited liability underlying assets. (Thus zero must be an absorbing barrier for the underlying stock.) Hence for a call option, $c(0, t)=0$. The contractual payoff function is $g(\cdot)$, meaning that at expiration time $T$, when the underlying

1 See Chapter 6 of Arnold (1992) for a discussion of Lipschitz and growth conditions.

2 American-type contingent claims, stochastic interest rates, and other extensions are considered in our ongoing work. 
price is $s$, the contingent claim contracts to pay $g(s)$ dollars. Therefore, to prevent arbitrage, $v(s, T)=g(s)$. We assume that the value of the claim can be expressed, using the Feynman-Kac Theorem, as the discounted expectation of its payoff under a risk-neutral probability measure. ${ }^{3}$ In the one-dimensional case

$$
v(s, t)=E\left\{e^{-\int_{t}^{T} r(\tau) d \tau} g\left(\xi_{T}^{s, t}\right)\right\},
$$

where $\xi_{\tau}^{s, t}$, which will be termed the risk-neutralized process, denotes the diffusion that at time $t$ starts at the level $s$, and then obeys the stochastic differential equation (SDE)

$$
d \xi_{\tau}=r(\tau) \xi_{\tau} d \tau+\sigma\left(\xi_{\tau}, \tau\right) \xi_{\tau} d B_{\tau}
$$

In the two-dimensional case, $v(s, y, T)=g(s)$, and by assumption, the price of volatility risk takes the form $\lambda(s, y, t)$. The time $t$ value of a contingent claim will then have the form $v(s, y, t)$.

\section{A. The Intuitive Link Between a Diffusion Process and Properties of Option Prices}

The following lemma provides the intuitive basis for the properties of contingent claim prices established in this section.

Lemma. (No-Crossing) In the one-dimensional case, $s^{\prime} \geq s^{\prime \prime}$ implies that, with probability $1, \xi_{\tau}^{s^{\prime}, t} \geq \xi_{\tau}^{s^{\prime \prime}, t}$ for all $\tau \geq t$.

Proof: According to (4), a fixed sample path (realization) of the Brownian motion $B_{\tau}$, determines, for each pair $(s, t)$, a unique sample path for the process $\xi_{\tau}^{s, t}$ (starting from the level $s$ at time $t$ ). Consider two such sample paths, $\xi_{\tau}^{s^{\prime}, t}$ and $\xi_{\tau}^{s^{\prime \prime}, t}$, where $s^{\prime} \geq s^{\prime \prime}$. (For simplicity, we use the same notation for a stochastic process and its sample paths; no confusion should arise.) The claim in the lemma is then that the $\xi_{\tau}^{s^{\prime}, t}$ sample path that starts out at the higher level $s^{\prime}$ never crosses strictly below the $\xi_{\tau}^{s^{\prime \prime}, t}$ sample path that starts out at the lower level $s^{\prime \prime}$. Suppose otherwise.

3 Rather than assuming some particular set of restrictions on the diffusion parameters in (1) and (2) known to be sufficient for the applicability of the Feynman-Kac Theorem, we prefer to implicitly consider the full set of diffusion parameters consistent with the Theorem. Note also that our assumption that the value of the claim can be expressed as in (3) should be read as: "can be correctly expressed." We are not, for example, assuming that we can price (by (3), or otherwise) a claim with $g(s)=1 / s$ when the underlying asset has a positive probability of $s_{T}=0$. 
Then the two sample paths, which are continuous (with probability 1) must have intersected (for the first time after $t$ ) at some time, $t^{\prime}$. But given the Markovian nature of $\xi$ in (4), the two sample paths will have become identical from time $t^{\prime}$ onward. Therefore, if one $\xi$ sample path starts higher than another, it remains higher. ${ }^{4}$ This is true for almost every sample path of $B_{\tau}$. We call this the no-crossing property of one-dimensional diffusions. I

The no-crossing property is illustrated in Figure 1. An immediate consequence of the lemma is that, in the one-dimensional diffusion setting, a claim's price inherits monotonicity from the contractual payoff function. For if $s^{\prime} \geq s^{\prime \prime}$, then by the no-crossing lemma, $\xi_{T}^{s^{\prime}, t} \geq \xi_{T}^{s^{\prime \prime}, t}$. Therefore, if $g(\cdot)$ is non-decreasing, then $g\left(\xi_{T}^{s^{\prime}, t}\right) \geq g\left(\xi_{T}^{s^{\prime \prime}, t}\right)$. This implies $E\left\{g\left(\xi_{T}^{s^{\prime}, t}\right)\right\} \geq E\left\{g\left(\xi_{T}^{s^{\prime \prime}, t}\right)\right\}$, and hence, by $(3), v\left(s^{\prime}, t\right) \geq v\left(s^{\prime \prime}, t\right)$.

The no-crossing property in the one-dimensional case, upon which the preceding demonstration of inherited monotonicity relies, requires that $\xi_{t}$, the risk-neutralized process for $s_{t}$, be both continuous and Markovian, namely, a diffusion (see Karlin and Taylor (1981, p. 157)). A stochastic process that is not a diffusion need not feature the no-crossing property, and contingent claims thereon need not exhibit inherited monotonicity. In fact, Section IV provides a number of such examples. Also, in contrast to the one-dimensional case, the no-crossing property need not hold in the two-dimensional case and Section IV shows that inherited monotonicity is not always satisfied in that setting.

\section{B. Sufficient Conditions for Inherited Monotonicity}

Theorem 1 bounds the slope of a contingent claim's price-in other words, the claim's delta-by the bounds on the slope of the claim's contractual payoff function. ${ }^{5}$

${ }^{4}$ Or else it intersects with the lower sample path and both merge onward, which can happen, for instance, at an absorbing boundary.

${ }^{5}$ For an interesting treatment of points of non-differentiability in $g(s)$ see Bick (1982). 
Theorem 1. Let the payoff function $g$ be differentiable on its domain. ${ }^{6}$ (i) Suppose $s_{t}$ follows a one-dimensional diffusion. Then, for all $s$ and $t$,

$$
\inf _{q} g_{1}(q) \leq v_{1}(s, t) \leq \sup _{q} g_{1}(q) .
$$

(ii) If $s_{t}$ follows a two-dimensional diffusion with the property that the drift and diffusion parameters of the risk-neutralized process for $y$ do not depend on $s$, then $v_{1}(s, y, t)$ is similarly bounded.

Proof: Given the condition in (i), the claim price can be expressed as in (3) and (4). For $s^{\prime} \geq s^{\prime \prime}$, the random variable $\mathcal{X}:=\xi_{T}^{s^{\prime}, t}-\xi_{T}^{s^{\prime \prime}, t}$ is non-negative by the no-crossing property. Furthermore, $E\{\mathcal{X}\}=e^{\int_{t}^{T} r(\tau) d \tau}\left(s^{\prime}-s^{\prime \prime}\right)$. For every $\xi$ sample path, $g\left(\xi_{T}^{s^{\prime \prime}, t}+\mathcal{X}\right)=g\left(\xi_{T}^{s^{\prime \prime}, t}\right)+$ $\mathcal{X} g_{1}(\psi) \geq g\left(\xi_{T}^{s^{\prime \prime}, t}\right)+\mathcal{X} \inf _{q} g_{1}(q)$, where $\psi \in\left(\xi_{T}^{s^{\prime \prime}, t}, \xi_{T}^{s^{\prime \prime}, t}+\mathcal{X}\right)$. Therefore,

$$
\begin{aligned}
v\left(s^{\prime}, t\right) & =E\left\{e^{-\int_{t}^{T} r(\tau) d \tau} g\left(\xi_{T}^{s^{\prime \prime}, t}+\mathcal{X}\right)\right\} \geq E\left\{e^{-\int_{t}^{T} r(\tau) d \tau} g\left(\xi_{T}^{s^{\prime \prime}, t}\right)\right\}+E\left\{e^{-\int_{t}^{T} r(\tau) d \tau} \inf _{q} g_{1}(q) \mathcal{X}\right\} \\
& =v\left(s^{\prime \prime}, t\right)+\inf _{q} g_{1}(q)\left(s^{\prime}-s^{\prime \prime}\right) ; \text { i.e., } \frac{v\left(s^{\prime}, t\right)-v\left(s^{\prime \prime}, t\right)}{s^{\prime}-s^{\prime \prime}} \geq \inf _{q} g_{1}(q) .
\end{aligned}
$$

Similarly, one can demonstrate that

$$
\frac{v\left(s^{\prime}, t\right)-v\left(s^{\prime \prime}, t\right)}{s^{\prime}-s^{\prime \prime}} \leq \sup _{q} g_{1}(q) .
$$

The proof of part (ii) consists of showing that the no-crossing property is satisfied under the assumed conditions, and then repeating the steps in part (i). The details are in the appendix.

\section{Sufficient Conditions for Inherited Convexity}

The work of Merton (1973), Cox and Ross (1976), and Jagannathan (1984), has established that, when the underlying asset's risk-neutralized price process is proportional, convexity (concavity) of the contractual payoff function implies convexity (concavity) of the contingent claim price. ${ }^{7}$

${ }^{6}$ Note that $g$ must still satisfy all the regularity conditions that justify the maintained assumption that the price of a contingent claim can be represented by the Feynman-Kac Integral. In the Appendix, Theorem 1 is generalized to the case where the payoff function $g$ has a left and a right derivative everywhere on its domain, where the two need not be equal, and where one of them may be plus infinity (as is the left derivative at a jump discontinuity upward, when the function is continuous on the right there) or minus infinity (as with a jump discontinuity downward). In particular, the generalization implies that the points, where the left and right derivatives of $g$ are not equal, do not matter. It also implies that a jump-discontinuity upwards (downwards) yields infinity (minus infinity) as the upper (lower) bound on $v_{1}(s, t)$.

7 A proportional one-dimensional diffusion for the risk-neutralized process implies a deterministic volatility function; i.e., a Black-Scholes setting. 
The next theorem states that the condition that the underlying price be a diffusion is also sufficient for the contingent claim price to inherit convexity (concavity) from the contractual payoff function.

Theorem 2. Suppose that $s_{t}$ is either (i) a one-dimensional diffusion, or (ii) a two-dimensional diffusion featuring the twin properties: (a) the drift and diffusion parameters of the risk-neutralized process for $y_{t}$ do not depend on the level of $s_{t}$, and (b) the covariance between instantaneous percent changes in $s_{t}$, and changes in $y_{t}$ does not depend on the level of $s_{t}$. Then, if a claim's contractual payoff function is convex (concave), the claim price is convex (concave) as a function of the concurrent underlying asset price.

Proof: See the Appendix.

The method of proof is analogous to that of Theorem 1 and proceeds by combining the Feynman-Kac Theorem and a 'no-crossing' property of the relevant SDE's. An alternate geometric proof, based on the stochastic maximum principle, is available upon request. Condition (ii) of Theorem 2 is more restrictive than condition (ii) of Theorem 1. To guarantee inherited convexity we require the additional restriction, condition (ii)(b), that the instantaneous covariance between percent changes in $s$ and changes in $y$ does not depend on $s$. The instantaneous covariance between percent changes in $s$ and changes in $y$ is given by $\sigma(s, y, t) \theta(s, y, t) \rho(s, y, t)$. Condition (ii)(a) already requires that the function $\theta(\cdot)$ not depend on $s$. Hence the additional restriction on the instantaneous covariance imposed by (ii)(b) is that the product $\sigma(s, y, t) \rho(s, y, t)$ does not depend on $s$. This could occur in three ways. First, and pathologically, both $\sigma(\cdot)$ and $\rho(\cdot)$ may depend on $s$, but in such inverse ways that their product does not. Second, $\rho(\cdot)$ may be zero for all $s, y$ and t. Finally, both $\sigma(\cdot)$ and $\rho(\cdot)$ may not be dependent on $s$. In this third case, the risk-neutralized process for $s$ is a proportional stochastic process. ${ }^{8}$

Theorem 2 continues to apply when for all $\tau \in[t, T]$, the underlying asset pays a continuous proportional dividend at the rate $\delta^{\mathcal{U}}(\tau)$ and the contingent claim pays a continuous proportional

8 In an appendix to Merton (1973), B. Goldman provides an example where the payoff function is convex, but the call-price function is not. It then follows from Theorem 2, that the terminal stock price distribution assumed in that example cannot be generated by either a one-dimensional diffusion process or a two-dimensional diffusion satisfying condition (ii) of the Theorem. 
dividend at the rate $\delta^{\mathcal{O}}(\tau) .{ }^{9}$ In that case, Theorem 1 [case (ii)] takes the form:

$$
e^{\int_{t}^{T}\left(\delta^{\mathcal{O}}(\tau)-\delta^{2 t}(\tau)\right) d \tau} \inf _{q} g_{1}(q) \leq v_{1}(s, y, t) \leq e^{\int_{t}^{T}\left(\delta^{\mathcal{O}}(\tau)-\delta^{2 t}(\tau)\right) d \tau} \sup _{q} g_{1}(q)
$$

Case (i) takes the same form, but without the $y$.

\section{Sufficient Conditions for Inherited Bounds on the Elements of a Replicating Portfolio}

When a contingent claim can be replicated through a dynamic strategy of trading the underlying stock and other assets, the position in stock is given by $v_{1}(s, y, t)$. It follows then that the value of the non-stock position in a replicating portfolio is $v(s, y, t)-v_{1}(s, y, t) s$. Noting this, Theorem 1 can be reinterpreted as stating that the stock position prior to maturity is bounded by the bounds on the stock position at maturity. Likewise, the next theorem establishes sufficient conditions for the value of the non-stock position to be similarly bounded.

Theorem 3. Let the payoff function $g$ be differentiable on its domain. ${ }^{10}$ (i) If $s_{t}$ follows a one-dimensional diffusion, then for all $s$ and $t$,

$$
e^{-\int_{t}^{T} r(\tau) d \tau} \inf _{q}\left[g(q)-g_{1}(q) q\right] \leq v(s, t)-v_{1}(s, t) s \leq e^{-\int_{t}^{T} r(\tau) d \tau} \sup _{q}\left[g(q)-g_{1}(q) q\right]
$$

(ii) If $s_{t}$ is a two-dimensional diffusion satisfying the twin properties of case (ii) of Theorem 2 , then $v(s, y, t)$ is similarly bounded.

Proof: See the Appendix.

\section{Monotonicity, Convexity and the Pricing and Replication of Call Options}

In Section I, conditions are established, under which certain properties of a quite general contingent claim's price and its replicating portfolio are inherited from the corresponding properties of the contractual payoff function. In this section we examine the specific implications of these results for the pricing and replication of call options. Throughout, we assume that the limited

9 Theorem 2 need not be applicable when dividends are non-proportional. For example, consider a zero exercise price European call written on a stock paying a continuous version of the non-proportional dividend discussed in footnote 16 of Chapter 4 of Cox and Rubinstein (1985).

10 Like Theorem 1, Theorem 3 can be generalized to the case where the payoff function $g$ has a left and a right derivative everywhere on its domain, where the two need not be equal, and where one of them may be plus infinity or minus infinity. 
liability underlying asset, which pays no dividends, follows a one-dimensional diffusion, that interest rates are deterministic, and that a call price, $c(s, t)$, is given by the solution of the p.d.e.,

$$
r(t) c_{1}(s, t) s-r(t) c(s, t)+c_{2}(s, t)+\frac{1}{2}[\sigma(s, t) s]^{2} c_{11}(s, t)=0,
$$

subject to the terminal condition $c(s, T)=\max [0, s-K]$.

\section{A. Relations Between a Call's Replicating Portfolio and the Underlying Asset Price}

A call option, like any contingent claim in the current setting, is replicated by a dynamic strategy which, at time $t$, when the stock price is $s$, maintains $c_{1}(s, t)$ shares of the stock and $c(s, t)-c_{1}(s, t) s$ dollars in bonds. Propositions 1 and 2 establish that the replicating portfolio consists of a levered position in the stock. Propositions 1 and 2 also provide bounds on the stock and bond positions in the replicating portfolio and, in addition, show how those positions change with the value of the underlying stock.

Proposition 1. If the underlying asset price follows a one-dimensional diffusion, then (i) the stock position in a call's replicating portfolio is always long, but never by more than one share, and (ii) the stock position is non-decreasing in $s$. For all $s$ and $t$ such that $\max \left[0, s-K e^{-\int_{t}^{T} r(\tau) d \tau}\right]<c(s, t)<s$, the stock position is strictly increasing in $s$.

Proof: The bounds in Theorem 1 apply to any contingent claim. For a call option they become $0 \leq c_{1}(s, t) \leq 1$, which proves (i). Theorem 2 similarly implies $c_{11}(s, t) \geq 0$, and the non-decreasing claim in part (ii) is established. The appendix contains a proof of the "strictly increasing" claim in part (ii).

Proposition 2. If the underlying asset follows a one-dimensional diffusion, then (i) a call's replicating portfolio consists of a levered position in stock, with the amount of borrowing being no greater than $e^{-\int_{t}^{T} r(\tau) d \tau} K$; (ii) the amount of borrowing is non-decreasing in $s$; and (iii) the call's elasticity, $\Omega(s, t)$, satisfies $1 \leq \Omega(s, t) \leq 1+e^{-\int_{t}^{T} r(\tau) d \tau} K / c(s, t)$.

Proof: From Theorem 3,

$$
-e^{-\int_{t}^{T} r(\tau) d \tau} K \leq c(s, t)-c_{1}(s, t) s \leq 0
$$


which proves part (i). From Theorem 2,

$$
\frac{\partial\left[c(s, t)-c_{1}(s, t) s\right]}{\partial s}=-s c_{11}(s, t) \leq 0
$$

and, since $s \geq 0$, part (ii) is established. Rearranging the bounds on the bond position gives the part (iii) bounds on the call's elasticity:

$$
1 \leq \Omega(s, t)=\frac{c_{1}(s, t) s}{c(s, t)} \leq 1+e^{-\int_{t}^{T} r(\tau) d \tau} K / c(s, t)
$$

One immediate implication of Propositions 1 and 2 is that, since in any one-dimensional diffusion setting a call option is equivalent to a levered position in the stock, the absolute value of the risk premium on a call always exceeds that on the underlying stock in that setting. ${ }^{11}$

\section{B. The Relation Between a Call's Price and the Passage of Time}

A call's convexity, coupled with its implicit leverage in any one-dimensional diffusion setting, causes a call option to always be a wasting asset in the one-dimensional case. This is shown next.

Proposition 3. If the underlying asset follows a one-dimensional diffusion, then for all $s$ and $t, c_{2}(s, t) \leq 0$. For all $s$ and $t$ such that either (i) $\max \left[0, s-e^{-\int_{t}^{T} r(\tau) d \tau} K\right]<c(s, t)<s$ and $\sigma(s, t)>0$, or (ii) $0<c(s, t)<s$ and $r(t)>0$, a call is a strictly wasting asset, i.e., $c_{2}(s, t)<0$.

Proof: $\quad$ Rewriting the p.d.e. in (5) gives

$$
c_{2}(s, t)=-\frac{1}{2}[\sigma(s, t) s]^{2} c_{11}(s, t)-r(t) c(s, t)(\Omega(s, t)-1)
$$

Theorem 2 implies that the first term on the RHS of (6) is non-positive for all $s$ and $t$. Proposition 2 implies that $\Omega(s, t) \geq 1$. Since $r(t) \geq 0$ for all $t$, the second term on the RHS of $(6)$ is non-positive for all $s$ and $t$. Since the RHS of (6) is non-positive, so is the LHS of (6).

11 Grundy (1991) shows that option prices contain information not only about the riskneutralized distribution of the underlying asset, but also about its true distribution, provided the underlying asset follows a one-dimensional diffusion and the risk premium on the option can be bounded. Proposition 1 has established that the particular bound examined in that paper is always satisfied for a one-dimensional diffusion. 
Turning to the strong inequality claim, we have from the strict convexity result of Proposition 1 that for all $s$ and $t$ such that $\max \left[0, s-K e^{-\int_{t}^{T} r(\tau) d \tau}\right]<c(s, t)<s$, the first term on the RHS of $(6)$ is strictly negative provided $\sigma(s, t)>0$. For $0<c(s, t)<s$, Proposition 1 implies that

$$
c(s, t)=c(0, t)+\int_{0}^{s} c_{1}(x, t) d x=\int_{0}^{s} c_{1}(x, t) d x<\int_{0}^{s} c_{1}(s, t) d x=c_{1}(s, t) s .
$$

Therefore $\Omega(s, t)=\frac{c_{1}(s, t) s}{c(s, t)}>1$. Thus, when $0<c(s, t)<s$ and $r(t)>0$, the second term on the RHS of $(6)$ is also strictly negative.

Propositions 1, 2 and 3 extend results familiar from a Black-Scholes setting (deterministic volatility) to any one-dimensional diffusion setting. ${ }^{12}$ While these results are intuitive, it is important to recognize that they need not be true when the underlying price does not follow a one-dimensional diffusion. It is shown in Section IV that if the underlying price process is either a multi-dimensional diffusion, or non-Markovian, or discontinuous, then it can be that in some $s$ range, either $c_{1}(s, t)<0$ holds or $c_{11}(s, t)<0$ holds, or both hold. When $c_{1}(s, t)<0$, a call's replicating portfolio will be short stock and long bonds. When $c_{11}(s, t)<0$, replication requires reducing the position in stock as the underlying price rises. Section IV also shows that when $c_{1}(s, t)<0$ or $c_{11}(s, t)<0$, a call can be a 'bloating' asset.

\section{The Relation Between a Call's Delta and its Exercise Price}

Proposition 4. If the underlying asset follows a one-dimensional diffusion, then $c_{1}(s, t)$, or the call's delta, does not increase with the exercise price. If $c_{1}(s, t)$ is also differentiable w.r.t. the exercise price $K$, then $\frac{\partial^{2} c}{\partial K \partial s} \leq 0$.

Proof: Consider a bullish money spread; long a call with exercise price $K_{1}$, and short a call with a larger exercise price $K_{2}$. The minimum derivative of this spread's final payoff function is zero. Denote the price of the spread by $M(s, t):=c\left(s, t ; K_{1}\right)-c\left(s, t ; K_{2}\right)$. Then, by Theorem 1 , at any time before expiration, $M_{1}(s, t) \geq 0$. In other words, $c_{1}\left(s, t ; K_{1}\right)-c_{1}\left(s, t ; K_{2}\right) \geq 0$.

12 In the deterministic volatility setting of Black-Scholes, it is also well known that for all $s$ and $t, \Omega_{1}(s, t)<0$ and $\Omega_{2}(s, t)>0$. These two properties do not necessarily generalize to a onedimensional diffusion with non-deterministic volatility. Still, the two inequalities will be satisfied for some sufficiently large $s$ and sufficiently large $(T-t)$. 
Note that a (one-dimensional) diffusion underlying price process is only a special sufficient condition for the result in Proposition 4. A more general sufficient condition is that monotonicity be inherited from the contractual payoff function. Thus, by the Cox and Ross (1976) result, the exercise price proposition is true also for proportional underlying processes, even when those are not diffusions.

We have considered the implications of Theorems 1, 2, and 3 for call options, but clearly, similar implications apply to put options, as well. For example, it is an immediate corollary of Theorem 1 that a put's delta is bounded between 0 and -1 . Similarly, by Theorem 2, a put price is always a convex function of the underlying asset. The following call option results also apply to put options with the appropriate modifications.

\section{The Comparative Statics of Interest Rates, Dividends, and Volatility}

We continue to assume that the underlying, limited-liability asset follows a one-dimensional diffusion, and that interest rates are deterministic.

\section{A. The Comparative Statics of Interest Rate Changes}

We wish to compare the prices of call options across two economies. In economy $\mathcal{A}$ the interest rate is $r^{\mathcal{A}}(t)$. The interest rate in an otherwise equivalent economy $\mathcal{B}$ is $r^{\mathcal{B}}(t)$. The underlying asset pays no dividends prior to the option's expiration.

\section{A.1. An upward shift in the term structure increases call prices}

Theorem 4. Consider two economies, $\mathcal{A}$ and $\mathcal{B}$, differing in their instantaneous interest rates, $r^{\mathcal{A}}(\tau)$ and $r^{\mathcal{B}}(\tau)$. Suppose that for all $\tau \in[t, T], r^{\mathcal{B}}(\tau) \geq r^{\mathcal{A}}(\tau)$ and $\int_{t}^{T} r^{\mathcal{B}}(\tau) d \tau>\int_{t}^{T} r^{\mathcal{A}}(\tau) d \tau$. Then, for all $s$ and $t, c^{\mathcal{B}}(s, t)$, the price of a call in economy $\mathcal{B}$ expiring at $T$, is at least as large as $c^{\mathcal{A}}(s, t)$, the price of an otherwise equivalent call in economy $\mathcal{A}$. For all $s$ and $t$, if $0<c^{\mathcal{B}}(s, t)<s$, then $c^{\mathcal{B}}(s, t)>c^{\mathcal{A}}(s, t)$.

Proof: See the Appendix. 


\section{A.2. A decrease in $e^{-\int_{t}^{T} r(\tau) d \tau} K$ can decrease call prices}

It is important to recognize what we have not established in Theorem 4 . In order to guarantee that $c^{\mathcal{B}}\left(s_{t}, t\right)>c^{\mathcal{A}}\left(s_{t}, t\right)$ it is not enough that the term structures differ across the two economies in such a way that the time $t$ value of a riskless bond maturing at time $T$ is smaller in economy $\mathcal{B}$. Theorem 4 requires not only that $\int_{t}^{T} r^{\mathcal{B}}(\tau) d \tau>\int_{t}^{T} r^{\mathcal{A}}(\tau) d \tau$, but, in addition, that $r^{\mathcal{B}}(\tau) \geq r^{\mathcal{A}}(\tau)$ for all $\tau \in[t, T] .{ }^{13}$ Consider the following two otherwise equivalent economies. In economy $\mathcal{A}$ the interest rate is given by the step-down function

$$
r^{\mathcal{A}}(\tau)= \begin{cases}\mathcal{R}, & \text { for } \tau \in\left[t, T-\frac{1}{2}(T-t)\right] \\ 0, & \text { for } \tau \in\left(T-\frac{1}{2}(T-t), T\right]\end{cases}
$$

In economy $\mathcal{B}$ the interest rate is given by the step-up function

$$
r^{\mathcal{B}}(\tau)= \begin{cases}0, & \text { for } \tau \in\left[t, T-\frac{1}{2}(T-t)\right] \\ \mathcal{R}, & \text { for } \tau \in\left(T-\frac{1}{2}(T-t), T\right] .\end{cases}
$$

Note that

$$
\int_{t}^{T} r^{\mathcal{A}}(\tau) d \tau=\frac{T-t}{2} \mathcal{R}=\int_{t}^{T} r^{\mathcal{B}}(\tau) d \tau
$$

Suppose that the underlying asset follows a diffusion of the form

$$
d s_{\tau}=r(\tau) s_{\tau} d \tau+\sigma\left(s_{\tau}, \tau\right) s_{\tau} d B_{\tau}
$$

where the volatility depends on the underlying asset price and time in the following way: For some $H>0$ and some strictly non-zero $\varpi(s, \tau)$,

$$
\sigma(s, \tau)= \begin{cases}\varpi(s, \tau), & \text { if } s>H \text { and } \tau \in\left[t, T-\frac{T-t}{2}\right] \\ 0, & \text { otherwise. }\end{cases}
$$

Now suppose that $s_{t}^{\mathcal{A}}=s_{t}^{\mathcal{B}}=s_{t}$ and that $s_{t}, H$, and $K$ are such that

$$
s_{t}<H<e^{\mathcal{R} \frac{T-t}{2}} s_{t}<K .
$$

13 If $\int_{t}^{T} r^{\mathcal{B}}(\tau) d \tau>\int_{t}^{T} r^{\mathcal{A}}(\tau) d \tau$ and volatility is deterministic, then $c^{\mathcal{B}}\left(s_{t}, t\right)>c^{\mathcal{A}}\left(s_{t}, t\right)$. Only the integral, $\int_{t}^{T} r(\tau) d \tau$, enters the Black-Scholes formula. 
In economy $\mathcal{A}, s_{\tau}^{\mathcal{A}}$ will grow deterministically at the interest rate $\mathcal{R}$ until, at time $t+\ln \left(H / s_{t}\right) / \mathcal{R}$, the underlying asset price reaches the level $H$. After that time, $s_{\tau}^{\mathcal{A}}$ will follow the diffusion

$$
d s_{\tau}^{\mathcal{A}}= \begin{cases}\mathcal{R} s_{\tau}^{\mathcal{A}} d t+\varpi\left(s_{\tau}^{\mathcal{A}}, \tau\right) s_{\tau}^{\mathcal{A}} d B_{\tau}, & \text { for } \tau \in\left(t+\ln \left(H / s_{t}\right) / \mathcal{R}, T-\frac{T-t}{2}\right] \\ 0, & \text { for } \tau \in\left(T-\frac{T-t}{2}, T\right] .\end{cases}
$$

A possible sample path for $s_{\tau}^{\mathcal{A}}$ is depicted in Figure 2. With positive probability $s_{T}^{\mathcal{A}}>K$, and hence $c^{\mathcal{A}}\left(s_{t}, t\right)>0$.

In economy $\mathcal{B}, s_{\tau}^{\mathcal{B}}$ will remain equal to $s_{t}$ until time $T-\frac{T-t}{2}$. After time $T-\frac{T-t}{2}, s_{\tau}^{\mathcal{B}}$ will grow deterministically at the rate $\mathcal{R}$ until it reaches the level $s_{t} e^{\mathcal{R} \frac{T-t}{2}}$ at the option's maturity. The sample path for $s_{\tau}^{\mathcal{B}}$ is also depicted in Figure $2 \mathrm{~b}$. With certainty $s_{T}^{\mathcal{B}}<K$, and hence $c^{\mathcal{B}}\left(s_{t}, t\right)=0$.

Thus, in this setting, which is not a Black-Scholes setting, shifts in the term structure that leave the current price of a bond maturing at the option's expiration unaffected can affect the option's price. In this example, simply 'reordering' the interest rate profile affects option prices.

\section{B. The Comparative Statics of Dividend Rate Changes}

The next theorem compares the price of a call on an asset that pays dividends to the price of a similar call on an asset that does not.

Theorem 5. Consider two one-dimensional-diffusion priced underlying assets (traded in the same economy with the same deterministic interest rate dynamics) such that, for all $\tau \in[t, T]$, asset $\mathcal{A}$ pays a dividend at the rate $\delta(\tau) \geq 0$, with $\int_{t}^{T} \delta(\tau) d \tau>0$, while asset $\mathcal{B}$ pays no dividends prior to time $T$. Suppose that $\sigma^{\mathcal{A}}(s, t)=\sigma^{\mathcal{B}}(s, t)$ for all $s$ and $t$. Then $c^{\mathcal{A}}(s, t) \leq e^{-\int_{t}^{T} \delta^{\mathcal{A}}(\tau) d \tau} c^{\mathcal{B}}(s, t)$, for all $s$ and $t$. This inequality becomes strict when $0<c^{\mathcal{B}}(s, t)<s$.

Proof: See the Appendix.

Not surprisingly, given $s_{t}^{\mathcal{A}}=s_{t}^{\mathcal{B}}$, a call on the asset that pays dividends is no more valuable than a call on the asset that does not. What is less obvious, is that one can place an upper bound on the relative prices of the two calls purely in terms of the fraction of the dividend-paying stock's price that is due to dividends to be paid beyond the call's expiration. When dividends are paid at the continuous rate $\delta(\tau)$, the present value at time $t$ of the stock price at time $T$ is $e^{-\int_{t}^{T} \delta(\tau) d \tau} s_{t}$. Equivalently, the time $t$ value of the dividend distributions beyond time $T$ is $e^{-\int_{t}^{T} \delta(\tau) d \tau} s_{t}$. 
The bound in Theorem 5 is familiar from a Black-Scholes setting. Given deterministic return volatility, the value of a call on asset $\mathcal{A}$ when $s_{t}^{\mathcal{A}}=s^{\prime}$ is equal to the value of an otherwise equivalent call written on the fraction $e^{-\int_{t}^{T} \delta(\tau) d \tau}$ of asset $\mathcal{B}$ when $s_{t}^{\mathcal{B}}=s^{\prime}$. In turn, such a call has the same value as an otherwise equivalent call written on one whole unit of asset $\mathcal{B}$ when $s_{t}^{\mathcal{B}}=e^{-\int_{t}^{T} \delta(\tau) d \tau} s^{\prime}$.

$$
c^{\mathcal{A}}(s, t)=c^{\mathcal{B}}\left(e^{-\int_{t}^{T} \delta(\tau) d \tau} \cdot s, t\right)
$$

From the strict convexity of call prices in a Black-Scholes setting, we then have

$$
c^{\mathcal{A}}(s, t)<e^{-\int_{t}^{T} \delta(\tau) d \tau} c^{\mathcal{B}}(s, t)
$$

In a setting where the return volatility depends on the (contemporaneous) stock price, the equality in (7) need no longer be satisfied. Still, as is shown in Theorem 5, the inequality in (8) is always satisfied in any one-dimensional diffusion setting.

\section{The Comparative Statics of Volatility Changes}

Jagannathan (1984) clarifies the relation between the value of a call and the riskiness of the underlying stock. Consider two stocks $\mathcal{A}$ and $\mathcal{B}$, but this time $\sigma^{\mathcal{A}}(s, \tau) \neq \sigma^{\mathcal{B}}(s, \tau)$ while, for all $\tau, \delta^{\mathcal{A}}(\tau)=\delta^{\mathcal{B}}(\tau)=0$. Given $s_{t}^{\mathcal{A}}=s_{t}^{\mathcal{B}}$, a sufficient condition for calls on stock $\mathcal{B}$ maturing at time $T$ to be at least as valuable as otherwise equivalent calls on stock $\mathcal{A}$, is that the risk-neutral

probability distribution of $s_{T}^{\mathcal{B}}$ is riskier than the risk-neutral probability distribution of $s_{T}^{\mathcal{A}}$ in the Rothschild-Stiglitz (1970) sense. ${ }^{14}$ We generalize and extend Jagannathan's results in the next two theorems.

Theorem 6. Let $\sigma^{\mathcal{B}}(s, t) \geq \sigma^{\mathcal{A}}(s, t)$ for all $s$ and $t$ with strict inequality in some region. Let $v^{\mathcal{B}}$ and $v^{\mathcal{A}}$ denote the respective prices of two contingent claims (not necessarily calls) with the same expiration date and identical, convex contractual payoff functions. Then for all $s$ and $t$, $v^{\mathcal{B}}(s, t) \geq v^{\mathcal{A}}(s, t)$.

$14 \tilde{Y}$ is riskier than $\widetilde{X}$ in the Rothschild-Stiglitz sense if

$$
\tilde{Y} \stackrel{d}{=} \tilde{X}+\widetilde{\varepsilon}, \quad \text { and } \quad E\{\tilde{\varepsilon} \mid X\}=0 \text { for all } X .
$$


Proof: See the Appendix.

As an example, if the volatility were always bounded by that of a particular CEV process, the call price would be bounded by the corresponding CEV option pricing model.

Theorem 7. Suppose that for all $s$ and $t, \sigma^{\mathcal{B}}(s, t) \geq \sigma^{\mathcal{A}}(s, t)$. For $s_{t}^{\mathcal{A}}=s_{t}^{\mathcal{B}}$, the risk-neutral distribution of $s_{T}^{\mathcal{B}}$ is riskier, in the Rothschild-Stiglitz sense, than the risk-neutral distribution of $s_{T}^{\mathcal{A}}$.

Proof: Let $\xi_{\tau}^{\mathcal{A}}{ }^{s, t}$ and $\xi_{\tau}^{\mathcal{B}}{ }^{s, t}$ be the time $\tau$ risk-neutralized prices of assets $\mathcal{A}$ and $\mathcal{B}$ satisfying

$$
d \xi_{\tau}^{\mathcal{A}}=r(\tau) \xi_{\tau}^{\mathcal{A}} d \tau+\sigma^{\mathcal{A}}\left(\xi^{\mathcal{A}}, \tau\right) \xi^{\mathcal{A}} d B_{\tau}
$$

and

$$
d \xi_{\tau}^{\mathcal{B}}=r(\tau) \xi_{\tau}^{\mathcal{B}} d \tau+\sigma^{\mathcal{B}}\left(\xi^{\mathcal{B}}, \tau\right) \xi^{\mathcal{B}} d B_{\tau}
$$

with a common initial condition, $s$, at time $t$.

Consider two contingent claims written, respectively, on the two assets, featuring the same convex payoff function $g(\cdot)$. By the Feynman-Kac representation, the prices of the claims are $v^{i}(s, t)=e^{-\int_{t}^{T} r(\tau) d \tau} E\left\{g\left(\xi_{T}^{i s, t}\right)\right\},(i=\mathcal{A}, \mathcal{B})$. For this setting, Theorem 6 has established that $v^{\mathcal{B}}(s, t) \geq v^{\mathcal{A}}(s, t)$. It then follows that $E\left\{g\left(\xi_{T}^{\mathcal{B}^{s, t}}\right)\right\} \geq E\left\{g\left(\xi_{T}^{\mathcal{A}^{s, t}}\right)\right\}$ for arbitrary convex $g(\cdot)^{\text {'s. }}$ This condition is equivalent to $\xi_{\tau}^{\mathcal{A}^{s, t}}$ being riskier than $\xi_{\tau}^{\mathcal{B}^{s, t}}$ in the Rothschild-Stiglitz sense.

D. Conditions under which Black-Scholes provides Bounds on Option Prices

Interesting special cases of Theorem 6 occur when, for all $s$ and $t$, either $\sigma^{\mathcal{B}}(s, t)=\bar{\sigma}(t) \geq$ $\sigma^{\mathcal{A}}(s, t)$, or $\sigma^{\mathcal{B}}(s, t) \geq \sigma^{\mathcal{A}}(s, t)=\underline{\sigma}(t)$. Let $c^{b s(\sigma)}(s, t)$ denote the Black-Scholes value of a call on a stock with deterministic volatility $\sigma(s, t)=\sigma(t)$ for all $s$ and $t$.

Theorem 8. If for all $s$ and $t, \sigma^{\mathcal{B}}(s, t)=\bar{\sigma}(t) \geq \sigma^{\mathcal{A}}(s, t)$, then $c^{\mathcal{A}}(s, t) \leq c^{b s(\bar{\sigma})}(s, t)$. If for all $s$ and $t, \sigma^{\mathcal{B}}(s, t) \geq \sigma^{\mathcal{A}}(s, t)=\underline{\sigma}(t)$, then $c^{\mathcal{B}}(s, t) \geq c^{b s(\underline{\sigma})}(s, t)$.

Proof: Theorem 8 is a special case of Theorem $6 .^{15}$

15 El Karoui, Jeanblanc-Picqué and Visvanathan provide an alternate proof of the special case result in Theorem 8. We thank Darrell Duffie for bringing this paper to our attention. Independent derivations of variants of Theorem 6 can be found in El Karoui, Jeanblanc-Picqué and Shreve (1995) and Avellaneda, Levy and Parás. 
Of major practical relevance to anyone charged with hedging an option position is that, despite a lack of knowledge of the functional form of the relation $\sigma(s, t)$, knowledge of bounds on that relation over the option's life, $\bar{\sigma}$ and $\underline{\sigma}$, provides bounds on the option's delta for any $s$ and t. These bounds are an immediate implication of Theorem 8 and the convexity property of option prices.

Proposition 5. If for all $s$ and $t, \underline{\sigma}(t) \leq \sigma(s, t) \leq \bar{\sigma}(t)$, then $c_{1}^{b s(\bar{\sigma})}\left(s^{\prime \prime}, t\right) \leq c_{1}(s, t) \leq c_{1}^{b s(\bar{\sigma})}\left(s^{\prime}, t\right)$, where $s^{\prime \prime}$ solves $c^{b s(\underline{\alpha})}(s, t)=c^{b s(\bar{\sigma})}\left(s^{\prime \prime}, t\right)+c_{1}^{b s(\bar{\sigma})}\left(s^{\prime \prime}, t\right)\left(s-s^{\prime \prime}\right)$ and $s^{\prime}$ solves $c^{b s(\underline{\sigma})}(s, t)=c^{b s(\bar{\sigma})}\left(s^{\prime}, t\right)-$ $c_{1}^{b s(\bar{\sigma})}\left(s^{\prime}, t\right)\left(s^{\prime}-s\right)$.

Proof: As depicted in Figure 3, if the lower bound on delta were violated, then, even if the option took on its minimal possible value, convexity would imply that for values of the underlying asset less than $s^{\prime \prime}$, the option's value would violate its upper bound. Similarly, if the upper bound on delta were violated then, even if the option took on its minimal possible value, convexity would imply that for values of the underlying asset greater than $s^{\prime}$, the option's value would violate its upper bound.

When the option's value is known, the bounds on its delta can be strengthened as follows.

Proposition 6. If for all $s$ and $t, \sigma(s, t) \leq \bar{\sigma}(t)$, then for any $s$ and $t$ such that one knows $c(s, t)$, $c_{1}^{b s(\bar{\sigma})}\left(s^{\prime \prime}, t\right) \leq c_{1}(s, t) \leq c_{1}^{b s(\bar{\sigma})}\left(s^{\prime}, t\right)$, where $s^{\prime \prime}$ solves $c(s, t)=c^{b s(\bar{\sigma})}\left(s^{\prime \prime}, t\right)+c_{1}^{b s(\bar{\sigma})}\left(s^{\prime \prime}, t\right)\left(s-s^{\prime \prime}\right)$ and $s^{\prime}$ solves $c(s, t)=c^{b s(\bar{\sigma})}\left(s^{\prime}, t\right)-c_{1}^{b s(\bar{\sigma})}\left(s^{\prime}, t\right)\left(s^{\prime}-s\right)$.

Proof: The logic is the same as that of Proposition 5.

\section{Pricing when the Underlying Asset does not follow a One-Dimensional Diffusion}

Sections II and III examin the pricing of call options in a one-dimensional diffusion setting, and establish that much of the intuition familiar from the Black-Scholes model carries over to the case where the underlying asset's volatility depends on both the contemporaneous price and time. This section considers the many interesting settings in which call prices need not possess any of their familiar properties. In particular, we demonstrate that if the process describing changes in the value of the underlying asset is either discontinuous or non-Markovian, or the underlying asset 
follows a multi-dimensional diffusion, then replicating a call's payoff can involve shorting stock and lending, and selling additional stock as the stock price rises. Throughout these examples the 'no-crossing' property is violated.

\section{A. Call Prices in a Discontinuous Markovian Setting}

Suppose the underlying asset follows a non-proportional process, such that below a certain level $H$ it behaves like a mixed diffusion-jump process, ${ }^{16}$ and above $H$ it grows at the interest rate. Formally,

$$
d s_{t}=\left\{\begin{array}{cl}
r(t) s_{t} d t & \text { for } \quad s_{t} \geq H \\
(r(t)-\Lambda(J-1)) s_{t} d t+s_{t} d q_{t} & \text { for } \quad 0<s_{t}<H
\end{array}\right.
$$

where $q_{t}$ is a Poisson process governing jumps in the stock price, $\Lambda$ is the mean number of jumps per unit time, and $(J-1)$ is the percentage price increase in the stock, if the Poisson event occurs.

Possible stock price paths are depicted in Figure 4a. Consider two possible time $t$ stock prices, $s^{\prime}$ and $s^{\prime \prime}$, with $0<s^{\prime \prime}<H<s^{\prime}$. For a given realization of the random component of the process, the stock price path starting at $s^{\prime \prime}$ can 'jump through' the path starting at $s$ ' which is larger than $s^{\prime \prime}$. Continuity precludes this in the one-dimensional case; recall the 'no-crossing' property of Section IA. Now consider a call option on this asset with an exercise price $K$ and $s^{\prime} e^{\int_{t}^{T} r(\tau) d \tau}<K<s^{\prime \prime} J$. When $s_{t}=s^{\prime}$, the option will, with certainty, finish out-of-the-money. When $s_{t}=s^{\prime \prime}$, the option has a positive probability of finishing in-the-money. Thus we have

$$
0=c(0, t)<c\left(s^{\prime \prime}, t\right)>c\left(s^{\prime}, t\right)=0
$$

i.e., the call price is not everywhere increasing, and therefore cannot be everywhere convex, in the underlying asset price.

Another example of a Markovian underlying process for which call prices are non-increasing in the underlying price is depicted in Figure $4 \mathrm{~b} .{ }^{17}$ The non-recombining binomial tree depicted illustrates a setting where a firm's management faces the following incentive problem. Suppose

16 See Merton (1976) for the development of an option pricing model applicable when the underlying follows a proportional mixed diffusion-jump process with a diversifiable jump component.

17 For yet another example, see footnote 14 of Chapter 4 of Cox and Rubinstein (1985). 
that management will be evaluated on the basis of the stock price at date $T$ relative to some goal, $\mathcal{G}$. Failure to meet the benchmark level, $\mathcal{G}$, will result in termination. Exceeding the goal will bring forth a bonus. If at date $T-1$ the firm has done poorly and the stock price is low, say $s_{T-1}=s^{\prime \prime}$, the firm must switch to high variance projects in order for there to be any chance of meeting the benchmark necessary for management to retain their posts. Alternately, if the stock price is high at date $T-1$, say $s_{T-1}=s^{\prime}$, management can, and will, effectively lock in their future bonuses by switching to a low risk investment strategy. The 'no-crossing' property is violated.

Now consider the date $T-1$ value of a call option with a date $T$ maturity written on the stock of this company. When $t=T-1$, and $K$ is as depicted in Figure $4 \mathrm{~b}$, the call price again satisfies the set of relations in (9). Interestingly, if back at time $T-2$ the stock price is equal to $s^{\prime \prime \prime}$, then the replicating strategy at that time involves shorting the underlying stock and lending. Further, an increase in the interest rate from $T-2$ to $T-1$ will decrease the call's value. We now turn to a continuous but non-Markovian setting, which also fails to satisfy the 'no-crossing' property. $^{18}$

\section{B. Option Prices in a Continuous Non-Markovian Setting}

When the underlying asset's instantaneous volatility depends not only on the contemporaneous price and time, but also on past prices, the process is said to be retarded. ${ }^{19}$ While retarded processes are relatively unexplored in the derivatives literature, they arise quite naturally when the volatility of a stock reflects the underlying firm's investment and financing decisions. That a stock's volatility is related to the contemporaneous stock price as a reflection of the firm's investment and financing policy is familiar from the Displaced Diffusion Option Pricing Model of Rubinstein (1983) and the Compound Option Pricing Model of Geske (1979). In both these models

18 Figure $4 \mathrm{~b}$ can be viewed as depicting a continuous, but non-Markovian process. Suppose that between trading dates one could observe (but not trade along) the trajectory of prices. Now suppose that at time $\check{t}$, one observed a trajectory level of $\breve{s}$. One could not then characterize the distribution of $s_{T}$ given only the knowledge that $s_{\breve{t}}=\breve{s}$. One would also need to know a past stock price as well (e.g., whether $s_{T-1}$ was equal to $s^{\prime}$ or $s^{\prime \prime}$.)

19 For a discussion of such processes, see Mohammed (1978). 
the firm's financing and investment decisions predate the option's issue date. But when financing and investment decisions occur during the option's life, and adjustment costs are such that the optimal controls are not continuous functions of the underlying firm value at each instant in time but instead exhibit hysteresis, the stock price process can be non-Markovian. ${ }^{20,21}$

The time line below depicts the setting we have in mind. The investment-financing decision made at time $t^{\prime}$ will depend on the value of the firm at $t^{\prime}$ as proxied by $s_{t^{\prime}}$. For simplicity, we assume that prior to time $t^{\prime}$, the stock's volatility is a constant $\overline{\bar{\sigma}}$.

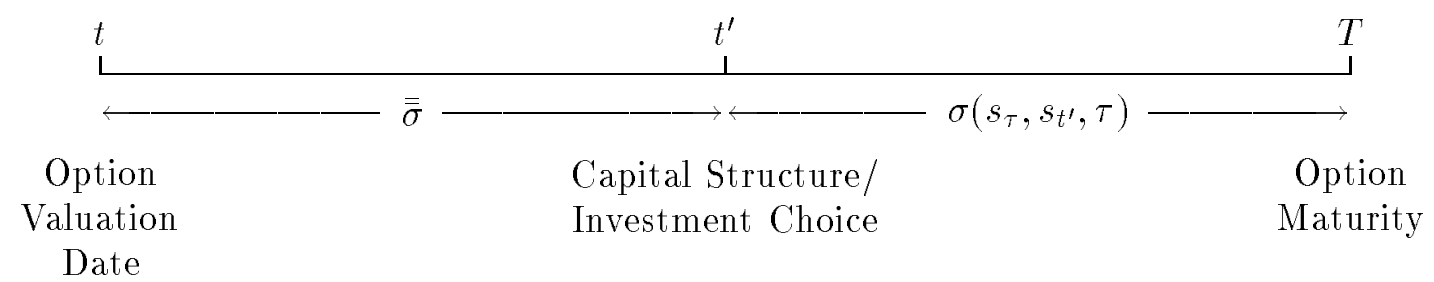

It is true that after $s_{t^{\prime}}$ has been realized at time $t^{\prime}$, it will be possible to represent the volatility of the stock at all times $\tau \in\left[t^{\prime}, T\right]$ as some function $\Theta\left(s_{\tau}, \tau\right)$. But the functional form of $\Theta(\cdot)$ cannot be determined ex ante. Ex ante, the volatility at all times $\tau \in\left[t^{\prime}, T\right]$ takes the form $\sigma\left(s_{\tau}, s_{t^{\prime}}, \tau\right)$. Ex ante, the process is non-Markovian.

As an analytically tractable example, consider an unlevered firm that will pay no dividends prior to time $T$ and will replace its assets at time $t^{\prime}$. Management will choose the replacement assets in a manner that reflects an incentive problem similar to that underlying Figure $4 \mathrm{~b}$. The lower the value of $s_{t^{\prime}}$, the higher the volatility of the replacement assets. Assume that for $\tau \in\left[t^{\prime}, T\right]$,

$$
\sigma\left(s_{\tau}, s_{t^{\prime}}, \tau\right)=\sigma\left(s_{t^{\prime}}\right)= \begin{cases}-\ln \left(s_{t^{\prime}} / \mathcal{G}\right) / \sqrt{T-t^{\prime}}, & \text { for } s_{t^{\prime}} \leq \mathcal{G} \\ 0, & \text { otherwise }\end{cases}
$$

20 For a formal model of optimal investment policy given the irreversibility of investment, see Dixit and Pindyck (1994). Fischer, Heinkel and Zechner (1989) model a firm's optimal dynamic capital structure choice given recapitalization costs.

21 That the firm faces adjustment costs is not inconsistent with our implicit assumption that the securities issued by the firm, and contingent claims thereon, are traded in frictionless capital markets. 
For $s_{t^{\prime}} \leq \mathcal{G}$, the firm chooses replacement assets that subsequently have constant volatility. The level of that constant volatility is a decreasing function of $s_{t^{\prime}}$, reaching zero for $s_{t^{\prime}}=\mathcal{G}$. For all $s_{t^{\prime}}>\mathcal{G}$, the firm chooses riskless replacement assets. For a given realization of the Brownian motion driving $s_{\tau}$, the risk-neutralized stock price path with initial condition $s^{\prime \prime}$ at time $t^{\prime}$ can potentially cross the path with initial condition $s^{\prime}>s^{\prime \prime}$.

Now consider the time $t^{\prime}$ value of a call option on this stock with a time $T$ expiration and an exercise price $K$ such that $e^{-\int_{t^{\prime}}^{T} r(w) d w} K=\mathcal{G}$. Since after $t^{\prime}$ the stock will have a constant volatility, the level of that volatility being determined by $s_{t^{\prime}}$ as in (10), we have

$$
c\left(s, t^{\prime}\right)=c^{b s\left(\sigma\left(s_{t^{\prime}}\right)\right)}\left(s, t^{\prime}\right)
$$

The function $c\left(s, t^{\prime}\right)$ is depicted in Figure 5a. The plot consists of the locus of points where a vertical line drawn from the $x$-axis at, say, the point $s^{\prime}$, intersects the dashed convex curve which plots $c^{b s\left(\sigma\left(s^{\prime}\right)\right)}\left(s, t^{\prime}\right)$. Notice how the vertical line drawn from $s^{\prime \prime}<s^{\prime}$ simultaneously intersects the locus and a second dashed convex curve that lies everywhere above $c^{b s\left(\sigma\left(s^{\prime}\right)\right)}\left(s_{t^{\prime}}, t^{\prime}\right)$. This higher convex curve plots $c^{b s\left(\sigma\left(s^{\prime \prime}\right)\right)}\left(s, t^{\prime}\right)$. From the volatility specification in (10) we have $\sigma\left(s^{\prime \prime}\right)>\sigma\left(s^{\prime}\right)$ since $s^{\prime \prime}<s^{\prime}$. For all $s_{t^{\prime}}>e^{-\int_{t^{\prime}}^{T} r(w) d w} K$ the locus of points is very easy to construct. With zero volatility in the future, the call is certain to finish-in-the-money, and is worth $s_{t^{\prime}}-e^{-\int_{t^{\prime}}^{T} r(w) d w} K$ at time $t^{\prime}$. The humped shape of the plot is determined by two opposing forces. As $s_{t^{\prime}}$ increases, the underlying stock becomes more valuable (which tends to increase the call price); but the underlying stock also becomes less volatile over the option's remaining life (which tends to decrease the call price). In a (Markovian) one-dimensional diffusion setting, Theorem 1 states that the first force is always the stronger. In contrast, in this non-Markovian setting the second force can overwhelm the first, thereby creating the hump.

When, as here, a call price can be decreasing and concave in $s$, a call can also have other properties quite different from those of a Black-Scholes setting. A call price can be increasing with the passage of time and a call price can decrease when interest rates increase. First consider the effect of the passage of time by comparing the call price at time $t^{\prime}$ to its price at some $t$ prior to $t^{\prime}$. 
We assume that for all $\tau \in\left[t, t^{\prime}\right)$, the stock's volatility is a constant $\overline{\bar{\sigma}}$. During the interval from $t$ to $t^{\prime}$ the call price satisfies the p.d.e.

$$
c_{2}(s, t)=-\frac{1}{2}[\overline{\bar{\sigma}} s]^{2} c_{11}(s, t)-r(t) c(s, t)(\Omega(s, t)-1) .
$$

When a call is increasing and convex in $s$ for all $s$ and $t$, the two terms on the right-hand-side of (11) are non-positive. Propositions 1 and 2 establish that $c_{11}(s, t) \geq 0$ and $\Omega(s, t)>1$ in a one-dimensional diffusion setting. But when, as here, the call is strictly decreasing and concave in $s$ over some region, both terms are strictly positive in that region. Thus the call can be a 'bloating' asset. Figure 5b illustrates the non-wasting nature of the call over some range of $s$, by depicting both $c(s, t)$ and $c\left(s, t^{\prime}\right)$ when $t^{\prime}-t=1$ year, $T-t^{\prime}=1$ year, $\overline{\bar{\sigma}}=30 \%$ per annum, $K=\mathcal{G}=\$ 3$, and $r(\tau)=10 \%$ per annum for all $\tau \in[t, T]$. Consider the effect on the call price of an increase in interest rates. Given the above parameter values, $c(s, t)=10.69 \notin$ when $s=\$ 1$. Suppose that the interest rate during the period from $t$ to $t^{\prime}$ increases from $10 \%$ to $11 \%$. This upward shift in the term structure will cause a decline in the call price to $10.38 \phi$.

\section{Call Prices and Stochastic Volatility}

Subsections $A$ and $B$ of IV provide illustrations of how the 'no-crossing' property can be violated when the underlying process is either discontinuous or non-Markovian. The proof of Theorem 1 establishes that the 'no-crossing' property is never violated in a one-dimensional diffusion setting. Theorem 1 also provides sufficient restrictions on the drift and diffusion parameters of a two-dimensional diffusion to guarantee that the "no-crossing' property remains satisfied when

volatility is stochastic. But when these restrictions are not met, the 'no-crossing' property can be violated in a diffusion setting, and a call price can be decreasing in the value of the underlying.

Recall from Section I the definition of a two-dimensional diffusion. Suppose that interest rates are zero and that the drift of the risk-neutralized process for $y$ is zero. The risk-neutralized processes for $s$ and $y$ are then given by:

$$
\begin{aligned}
& d s_{t}=\sigma\left(s_{t}, y_{t}, t\right) s_{t} d B_{t}^{1}, \text { and } \\
& d y_{t}=\theta\left(s_{t}, y_{t}, t\right) d B_{t}^{2} .
\end{aligned}
$$


One could think of $s$ and $y$ as the share prices of two firms in a duopoly whose competitive strategies and share prices reflect their market shares.

As depicted in Figure 6, if $s$ is high and $y$ is low, then $\sigma(s, y, t)=\theta(s, y, t)=0$. If $s$ is low and $y$ is low, then $\sigma(s, y, t)=0$ and $\theta(s, y, t)>0$. If $y$ is high, then $\sigma(s, y, t)>0$ and $\theta(s, y, t)>0$. Note that condition (ii) of Theorem 1 is not met in this example. In particular, whether $\theta\left(s_{t}, y_{t}, t\right)$ is positive or zero depends on the value of $s_{t}$.

With initial condition $\left\{s^{\prime}, y^{\prime}\right\}, s_{T}=s^{\prime}$. With initial condition $\left\{s^{\prime \prime}, y^{\prime}\right\}$, there is a positive probability that $s_{T}>s^{\prime}$ even though $s^{\prime \prime}<s^{\prime}$, and hence the "no-crossing' property is violated in this example. Now consider a call option with $K>s^{\prime}$. For this option $c\left(s^{\prime}, y^{\prime}, t\right)=0$ and $c\left(s^{\prime \prime}, y^{\prime}, t\right)>0$. Thus, over a range of $s$ values, the option price is decreasing in $s$, and cannot then be everywhere convex in $s$.

\section{Conclusions}

This paper examines the general properties of prices of European contingent claims. We show that when the underlying stock follows a one-dimensional diffusion and interest rates are deterministic, the stock position in the dynamic portfolio that replicates a contingent claim (equivalently, the claim's price slope or "delta") is bounded by the infimum and the supremum of that position at maturity. Similar bounds also hold for the bond position in the claim's replicating portfolio. If the claim's payoff at maturity is convex (concave) in the price of the underlying asset at expiration, then prior to expiration the stock position in a replicating portfolio is increasing (decreasing) in the underlying asset's price. The bounds on a claim's delta also apply in a multi-dimensional diffusion setting, provided that the drift and diffusion parameters of the risk-neutralized version of the process driving the stochastic changes in volatility are independent of the underlying asset price. With appropriate further restrictions, the inherited convexity result can also be extended to a multi-dimensional diffusion setting. In sum, under stipulated, quite general diffusion conditions, properties of the contractual payoff function, like monotonicity and convexity, are inherited by the contingent claim price (as a function of the underlying price) prior to expiration. 
The bounds and inherited convexity results allow us to undertake comparative static analyses of the effects of changes in interest rates, in dividend rates, and in volatility on the prices of call options in a one-dimensional diffusion setting. First, we show that it is only in a deterministic volatility (Black-Scholes) setting that a decrease in the present value of the exercise price necessarily implies an increase in a call price. In general, contingent claim prices are determined by the entire term structure of instantaneous interest rates through the claim's maturity date, and not merely by their integral. A twist in the term-structure that leaves the present value of a call's exercise price unchanged can change the call's value. Second, we develop a new bound on the relative values of call options on two underlying assets; one that pays dividends, another that does not, but which are otherwise equivalent. Third, we show that when the underlying asset's volatility is bounded above (below), then, whatever the functional form of the relation between volatility, time, and the contemporaneous stock price, the option's price is bounded above (below) by its Black-Scholes value calculated at the bounding volatility level. We also show how to incorporate bounds on the underlying asset's volatility into the determination of bounds on an option's delta. Finally, we undertake a comparative statics analysis of the relation between the exercise price of a call and its delta in a one-dimensional diffusion setting. We establish that the call's delta is always non-decreasing in its exercise price.

The bounds on a contingent claim's delta established in a one-dimensional diffusion setting, and in certain restricted stochastic volatility settings, are shown to be a reflection of the fact that, for a given realization of the Brownian motion driving the risk-neutralized stock price process, the realized value of the process at the claim's maturity date is increasing in its starting value. We dub this the "no-crossing' property. We demonstrate that if we relax either the continuity or Markovian properties inherent in a diffusion, or we consider an unrestricted stochastic volatility setting, then the 'no-crossing' property can be violated. It is then shown that the price of a call option can be decreasing or concave over some underlying price range, increasing in the passage of time, and decreasing in the level of interest rates. 


\section{Appendix}

\section{Proof of part (ii) of Theorem 1}

(Throughout the proof, superscripts ' 1 ' and '2' denote indices - not powers.) Using the Feynman-Kac Theorem the value of the contingent claim can be expressed as

$$
v(s, y, t)=E\left\{e^{-\int_{t}^{T} r(\tau) d \tau} g\left(\xi_{T}^{1 s, y, t}\right)\right\}
$$

where $\xi^{1, y, t}$ and $\xi^{2 s, y, t}$ solve the system of SDE's

$$
\begin{aligned}
& d \xi_{\tau}^{1}=r(\tau) \xi_{\tau}^{1} d \tau+\sigma\left(\xi_{\tau}^{1}, \xi_{\tau}^{2}, \tau\right) \xi_{\tau}^{1} d B_{\tau}^{1} \\
& d \xi_{\tau}^{2}=\left(\beta\left(\xi_{\tau}^{1}, \xi_{\tau}^{2}, \tau\right)-\lambda\left(\xi_{\tau}^{1}, \xi_{\tau}^{2}, \tau\right) \theta\left(\xi_{\tau}^{1}, \xi_{\tau}^{2}, \tau\right)\right) d \tau+\theta\left(\xi_{\tau}^{1}, \xi_{\tau}^{2}, \tau\right) d B_{\tau}^{2}
\end{aligned}
$$

with $d B_{\tau}^{1} d B_{\tau}^{2}=\rho\left(\xi_{\tau}^{1}, \xi_{\tau}^{2}, \tau\right) d \tau$ and initial conditions $s$ and $y$ at time $t$. The SDE's in $(A 1)$ describe the 'risk-neutralized' processes for $s$ and $y$.

The conditions in (ii) imply that there exist functions $G^{1}$ and $G^{2}$, such that:

$$
\begin{aligned}
& G^{1}\left(\xi^{2}, \tau\right)=\beta\left(\xi^{1}, \xi^{2}, \tau\right)-\lambda\left(\xi^{1}, \xi^{2}, \tau\right) \theta\left(\xi^{1}, \xi^{2}, \tau\right), \text { and } \\
& G^{2}\left(\xi^{2}, \tau\right)=\left[\theta\left(\xi^{1}, \xi^{2}, \tau\right)\right]^{2} .
\end{aligned}
$$

In this case, expression $(A 1)$ simplifies to

$$
\begin{aligned}
& d \xi_{\tau}^{1}=r(\tau) \xi_{\tau}^{1} d \tau+\sigma\left(\xi_{\tau}^{1}, \xi_{\tau}^{2}, \tau\right) \xi_{\tau}^{1} d B_{\tau}^{1} \\
& d \xi_{\tau}^{2}=G^{1}\left(\xi_{\tau}^{2}, \tau\right) d \tau+\sqrt{G^{2}\left(\xi_{\tau}^{2}, \tau\right)} d B_{\tau}^{2}
\end{aligned}
$$

Thus, for any given realization of the $B_{\tau}^{2}$ process, condition (ii) guarantees that the path followed by $\xi_{\tau}^{2}$ is independent of the initial condition for $\xi_{\tau}^{1}$. Now consider two paths for $\xi_{\tau}^{1}$ differing only in their initial conditions. Given the realization of the $B_{\tau}^{2}$ process, we construct each path for $\xi_{\tau}^{1}$ from innovations $d B_{\tau}^{1}$, with the innovations themselves constructed as $d B_{\tau}^{1}=\rho\left(\xi_{\tau}^{1}, \xi_{\tau}^{2}, \tau\right) d B_{\tau}^{2}+$ $\sqrt{1-\left[\rho\left(\xi_{\tau}^{1}, \xi_{\tau}^{2}, \tau\right)\right]^{2}} d B_{\tau}^{3}$, where $B_{\tau}^{3}$ is a standard Brownian motion independent of $B_{\tau}^{2}$. Suppose these two paths for $\xi_{\tau}^{1}$ met at some time $t^{\prime}$. Could they cross over? Consider the SDE in $\left(A 1 a^{\prime}\right)$. By construction, both paths for $\xi_{\tau}^{1}$ always share a common realization of the $\xi_{\tau}^{2}$ process and hence, 
must merge from time $t^{\prime}$ onward. Suppose $s^{\prime \prime}<s^{\prime}$. Starting from $\left\{\xi_{t}^{1}=s^{\prime}, \xi_{t}^{2}=y\right\}$, the subsequent level of $\xi_{T}^{1}$ attained at time $T$ is always at least as great as the level attained when the processes start from $\left\{s^{\prime \prime}, y\right\}$. The rest of the proof involves defining $\mathcal{X}:=\xi_{T}^{1, s^{\prime}, y, t}-\xi_{T}^{1, s^{\prime \prime}, y, t}$, and proceeding as in the proof of part (i).

\section{Generalization of Theorem 1}

Suppose that the payoff function $g$ has a left and a right derivative everywhere on its domain, where the two need not be equal, and where one of them may be plus infinity (as is the left derivative at a jump discontinuity upward, when the function is continuous on the right there) or minus infinity (as with a jump discontunity downward). This, of course, covers calls, puts, and digital options). Then Theorem 1 generalizes by replacing the double inequality by the following

$$
\inf _{q}\left[\min \left(g_{1}(q-), g_{1}(q+)\right)\right] \leq v_{1}(s, t) \leq \sup _{q}\left[\max \left(g_{1}(q-), g_{1}(q+)\right)\right],
$$

where, for example, $g_{1}(q+)$ stands for the right derivative of $g$ at $q$.

Proof: Suppose $s_{t}$ follows a one-dimensional diffusion. The call price can then be expressed as in (3) and (4). For $s^{\prime} \geq s^{\prime \prime}$, the random variable $\mathcal{X}:=\xi_{T}^{s^{\prime}, t}-\xi_{T}^{s^{\prime \prime}, t}$ is non-negative by the no-crossing property. Furthermore, $E\{\mathcal{X}\}=e^{\int_{t}^{T} r(\tau) d \tau}\left(s^{\prime}-s^{\prime \prime}\right)$. For every $\xi$ sample path, $g\left(\xi_{T}^{s^{\prime \prime}, t}+\mathcal{X}\right) \geq g\left(\xi_{T}^{s^{\prime \prime}, t}\right)+\mathcal{X} \min \left(g_{1}(\psi-), g_{1}(\psi+)\right) \geq g\left(\xi_{T}^{s^{\prime \prime}, t}\right)+\mathcal{X} \inf _{q}\left[\min \left(g_{1}(q-), g_{1}(q+)\right)\right]$, where $\psi \in\left(\xi_{T}^{s^{\prime \prime}, t}, \xi_{T}^{s^{\prime \prime}, t}+\mathcal{X}\right)$. Here, we used the generalization of the Intermediate Value Theorem of differential calculus to the case where only the left and the right derivatives are guaranteed to exist. The rest of the proof follows the same steps as in the main text.

\section{The Cauchy Problem and the Proof of Theorem 2}

The Theorem 2 condition that the claim's payoff function is convex (concave) implies that the payoff function is continuous. First consider the Cauchy Problem: For given $T>0$, we wish to find $f \in C^{2,1}\left(\mathrm{IR}^{N} \times[0, T)\right)$ solving

$$
\mathcal{D} f(x, t)-R(x, t) f(x, t)+h(x, t)=0, \quad(x, t) \in \mathbb{R}^{N} \times[0, T),
$$

with the terminal condition 


$$
f(x, T)=g(x), \text { almost everywhere on } \mathrm{IR}^{N},
$$

and

$$
\mathcal{D} f(x, t)=f_{N+1}(x, t)+\sum_{i=1}^{N} f_{i}(x, t) \mu^{i}(x, t)+\frac{1}{2} \sum_{i=1}^{N} \sum_{j=1}^{N} \gamma^{i}(x, t) \gamma^{j}(x, t) \eta^{i j}(x, t) f_{i j}(x, t),
$$

and where $f_{N+1}(x, t)$ denotes the partial of $f$ with respect to $t$, and, for $i=1, \ldots, N, f_{i}(x, t)$ denotes the partial of $f$ with respect to the $i$ th element of the vector $x, R: \mathbb{R}^{N} \times[0, T] \rightarrow[0, \infty)$, $h: \mathbb{R}^{N} \times[0, T] \rightarrow \mathbb{R}, g: \mathbb{R}^{N} \rightarrow \mathbb{R}$, the functions $R, h$ and $g$ are continuous, and $\mu(x, t)$ is an $N \times 1$ vector whose $i$ th element $\mu^{i}(x, t)$ is such that $\mu^{i}: \mathbb{R}^{N} \times[0, T] \rightarrow \mathbb{R}$, and the superscripts on $\gamma^{i}(x, t) \gamma^{j}(x, t) \eta^{i j}(x, t)$ do not denote powers but are instead indices. $\gamma(x, t)$ is an $N \times 1$ vector whose $i$ th element $\gamma^{i}(x, t): \mathbb{R}^{N} \times[0, T] \rightarrow \mathbb{R}$, for $i=1, \ldots, N$. Each function $\eta^{i j}(x, t)=\eta^{j i}(x, t)$ : $\mathrm{IR}^{N} \times[0, T] \rightarrow \mathrm{IR}$, for all $i, j=1, \ldots, N$.

The Feynman-Kac solution to (A2)-(A4), when it exists, is given by

$$
f(x, t)=E\left\{\int_{t}^{T} \varphi_{t, \tau} h\left(\xi_{\tau}^{x, t}, \tau\right) d \tau+\varphi_{t, T} g\left(\xi_{T}^{x, t}\right)\right\},
$$

where $\varphi_{t, \tau}=e^{-\int_{t}^{\tau} R\left(\xi_{w}^{x, t}, w\right) d w}$. The elements, $\xi^{i x, t}$, of the $N \times 1$ vector $\xi^{x, t}$ solve the system of SDE's

$$
d \xi_{\tau}^{i}=\mu^{i}\left(\xi_{\tau}, \tau\right) d \tau+\gamma^{i}\left(\xi_{\tau}, \tau\right) d B_{\tau}^{i}
$$

with initial condition $x$ at time $t$. In addition, $d B_{\tau}^{i} d B_{\tau}^{j}=\eta^{i j}\left(\xi_{\tau}, \tau\right) d \tau$.

As an example suppose the underlying asset follows the two dimensional diffusion given in (2), interest rates are deterministic, and the price of volatility risk is given by $\lambda(s, y, t)$. The value of a contingent claim is given by the solution of the p.d.e.,

$$
\begin{aligned}
& v_{3}(s, y, t)+v_{1}(s, y, t) r(t) s+v_{2}(s, y, t)(\beta(s, y, t)-\lambda(s, y, t) \theta(s, y, t))+\frac{1}{2} v_{11}(s, y, t)[\sigma(s, y, t) s]^{2} \\
& +\frac{1}{2} v_{22}(s, y, t)[\theta(s, y, t)]^{2}+v_{12}(s, y, t) \sigma(s, y, t) s \theta(s, y, t) \rho(s, y, t)-r(t) v(s, y, t)=0,
\end{aligned}
$$

subject to the terminal condition $v(s, y, T)=g(s)$. Let the superscripts on the functions $Y^{1}, \ldots, Y^{4}$ denote indices not powers, and define the functions as:

$$
\begin{aligned}
Y^{1}(s, y, t) & =\beta(s, y, t)-\lambda(s, y, t) \theta(s, y, t) . & Y^{3}(s, y, t) & =[\theta(s, y, t)]^{2} \\
Y^{2}(s, y, t) & =[\sigma(s, y, t) s]^{2} . & Y^{4}(s, y, t) & =\sigma(s, y, t) s \theta(s, y, t) \rho(s, y, t) .
\end{aligned}
$$


We can then rewrite (A6) as

$$
\begin{array}{r}
v_{3}(s, t)+v_{1}(s, y, t) r(t) s+v_{2}(s, y, t) Y^{1}(s, y, t)+\frac{1}{2} v_{11}(s, y, t) Y^{2}(s, y, t) \\
+\frac{1}{2} v_{22}(s, y, t) Y^{3}(s, y, t)+v_{12}(s, y, t) Y^{4}(s, y, t)-r(t) v(s, y, t)=0 .
\end{array}
$$

Taking the partial of (A7) with respect to $s$ gives

$$
\begin{gathered}
v_{13}(s, y, t)+v_{11}(s, y, t) r(t) s+v_{1}(s, y, t) r(t)+v_{12}(s, y, t) Y^{1}(s, y, t)+v_{2}(s, y, t) Y_{1}^{1}(s, y, t)+ \\
\frac{1}{2} v_{111}(s, y, t) Y^{2}(s, y, t)+\frac{1}{2} v_{11}(s, y, t) Y_{1}^{2}(s, y, t)+\frac{1}{2} v_{122}(s, y, t) Y^{3}(s, y, t)+ \\
\frac{1}{2} v_{22}(s, y, t) Y_{1}^{3}(s, y, t)+v_{112}(s, y, t) Y^{4}(s, y, t)+v_{12}(s, y, t) Y_{1}^{4}(s, y, t)-r(t) v_{1}(s, y, t)=0 .
\end{gathered}
$$

Let $f$ be the value of the first partial of a contingent claim's value with respect to the value of the underlying asset. The p.d.e. in (A8) can then be rewritten as

$$
\begin{gathered}
f_{3}(s, y, t)+f_{1}(s, y, t)\left[r(t) s+\frac{1}{2} Y_{1}^{2}(s, y, t)\right]+f_{2}(s, y, t)\left[Y^{1}(s, y, t)+Y_{1}^{4}(s, y, t)\right] \\
+\frac{1}{2} f_{11}(s, y, t) Y^{2}(s, y, t)+\frac{1}{2} f_{22}(s, y, t) Y^{3}(s, y, t)+f_{12}(s, y, t) Y^{4}(s, y, t) \\
+v_{2}(s, y, t) Y_{1}^{1}(s, y, t)+\frac{1}{2} v_{22}(s, y, t) Y_{1}^{3}(s, y, t)=0 .
\end{gathered}
$$

Expression (A9) is in the same form as (A2) with

$$
\begin{aligned}
h(x, t) & =v_{2}(s, y, t) Y_{1}^{1}(s, y, t)+\frac{1}{2} v_{22}(s, y, t) Y_{1}^{3}(s, y, t) . \\
N=2 . & \mu(x, t)=\left(\begin{array}{c}
r(t) s+\frac{1}{2} Y_{1}^{2}(s, y, t) \\
\left.Y^{1}(s, y, t)+Y_{1}^{4}(s, y, t)\right)
\end{array}\right) . \\
x=\left(\begin{array}{l}
s \\
y
\end{array}\right) . & \gamma(x, t)=\left(\begin{array}{ll}
\sqrt{Y^{2}(s, y, t)} \\
\sqrt{Y^{3}(s, y, t)}
\end{array}\right) . \\
R(s, t)=0 . & \text { if } i \neq j ; \\
\eta^{i j}(x, t) & = \begin{cases}\rho(s, y, t), & \text { otherwise. }\end{cases}
\end{aligned}
$$

Thus the Feynman-Kac solution for $v_{1}(s, y, t)$, when it exists, is given by

$$
\begin{aligned}
v_{1}(s, y, t)=E & \left\{\int _ { t } ^ { T } \left(v_{2}\left(\hat{\xi}_{\tau}^{1 s, y, t}, \hat{\xi}_{\tau}^{2 s, y, t}, \tau\right) Y_{1}^{1}\left(\hat{\xi}_{\tau}^{1 s, y, t}, \hat{\xi}_{\tau}^{2 s, y, t}, \tau\right)\right.\right. \\
& \left.\left.+\frac{1}{2} v_{22}\left(\hat{\xi}_{\tau}^{1 s, y, t}, \hat{\xi}_{\tau}^{2 s, y, t}, \tau\right) Y_{1}^{3}\left(\hat{\xi}_{\tau}^{1 s, y, t}, \hat{\xi}_{\tau}^{2 s, y, t}, \tau\right)\right) d \tau+g_{1}\left(\hat{\xi}_{T}^{1 s, y, t}\right)\right\},
\end{aligned}
$$


where $\hat{\xi}^{1 s, y, t}$ and $\hat{\xi}^{2 s, y, t}$ solve the system of SDE's

$$
\begin{aligned}
& d \hat{\xi}_{\tau}^{1}=\left(r(\tau) \hat{\xi}_{\tau}^{1}+\frac{1}{2} Y_{1}^{2}\left(\hat{\xi}_{\tau}^{1}, \hat{\xi}_{\tau}^{2}, \tau\right)\right) d \tau+\sqrt{Y^{2}\left(\hat{\xi}_{\tau}^{1}, \hat{\xi}_{\tau}^{2}, \tau\right)} d B_{\tau}^{1}, \\
& d \hat{\xi}_{\tau}^{2}=\left(Y^{1}\left(\hat{\xi}_{\tau}^{1}, \hat{\xi}_{\tau}^{2}, \tau\right)+Y_{1}^{4}\left(\hat{\xi}_{\tau}^{1}, \hat{\xi}_{\tau}^{2}, \tau\right)\right) d \tau+\sqrt{Y^{3}\left(\hat{\xi}_{\tau}^{1}, \hat{\xi}_{\tau}^{2}, \tau\right)} d B_{\tau}^{2},
\end{aligned}
$$

with initial condition $\{s, y\}$ at time $t$. In addition, $d B_{\tau}^{1} d B_{\tau}^{2}=\rho\left(\hat{\xi}^{1} \tau, \hat{\xi}_{\tau}^{2}, \tau\right) d \tau$. Note that these are not the SDE's describing the risk-neutralized processes for $s$ and $y$ given in $(A 1)$.

Condition (i) of Theorem 2 implies that there exists a function $Z$ such that

$$
Z(s, t)=\sigma(s, y, t) s .
$$

When the underlying asset follows a one-dimensional diffusion and condition (i) is satisfied, the Feynman-Kac solution for the first partial of the value of the contingent claim with respect to the value of the underlying asset then simplifies to

$$
v_{1}(s, t)=E\left\{g_{1}\left(\hat{\xi}_{T}^{s, t}\right)\right\}
$$

where $\hat{\xi}^{s, t}$ solves the SDE

$$
d \hat{\xi}_{\tau}=\left(r(\tau) \hat{\xi}_{\tau}+Z_{1}\left(\hat{\xi}_{\tau}, \tau\right)\right) d \tau+Z\left(\hat{\xi}_{\tau}, \tau\right) d B_{\tau}
$$

with initial condition $s$ at time $t$.

Suppose $s^{\prime}>s^{\prime \prime}$. Then by the 'no-crossing' lemma $\hat{\xi}_{T}^{s^{\prime}, t} \geq \hat{\xi}_{T}^{s^{\prime \prime}, t}$ with probability 1 . If for all $s, g_{11} \geq 0$ then $g_{1}$ is non-decreasing in $s$, and hence

$$
v_{1}\left(s^{\prime}, t\right)=E\left\{g_{1}\left(\hat{\xi}_{T}^{s^{\prime}, t}\right)\right\} \geq E\left\{g_{1}\left(\hat{\xi}_{T}^{s^{\prime \prime}, t}\right)\right\}=v_{1}\left(s^{\prime \prime}, t\right)
$$

Similarly, if for all $s, g_{11} \leq 0, v_{1}\left(s^{\prime}, t\right) \leq v_{1}\left(s^{\prime \prime}, t\right)$.

Now consider the two-dimensional case. Condition (ii) of Theorem 2 implies that there exist functions $G^{1}, G^{2}$ and $G^{3}$, where superscripts denote indices not powers, such that $G^{1}(y, t)=$ $Y^{1}(s, y, t), G^{2}(y, t)=Y^{3}(s, y, t)$ and $G^{3}(y, t) s=Y^{4}(s, y, t)$. The Feynman-Kac solution for the value of the first partial of the contingent claim with respect to the underlying asset then simplifies to

$$
v_{1}(s, y, t)=E\left\{g_{1}\left(\hat{\xi}_{T}^{1 s, y, t}\right)\right\}
$$


where $\hat{\xi}^{1 s, y, t}$ and $\hat{\xi}^{2 s, y, t}$ solve the system of SDE's

$$
\begin{aligned}
& d \hat{\xi}_{\tau}^{1}=\left(r(\tau) \hat{\xi}_{\tau}^{1}+\frac{1}{2} Y_{1}^{2}\left(\hat{\xi}_{\tau}^{1}, \hat{\xi}_{\tau}^{2}, \tau\right)\right) d \tau+\sqrt{Y^{2}\left(\hat{\xi}_{\tau}^{1}, \hat{\xi}_{\tau}^{2}, \tau\right)} d B_{\tau}^{1}, \\
& d \hat{\xi}_{\tau}^{2}=\left(G^{1}\left(\hat{\xi}_{\tau}^{2}, \tau\right)+G^{3}\left(\hat{\xi}_{\tau}^{2}, \tau\right)\right) d \tau+\sqrt{G^{2}\left(\hat{\xi}_{\tau}^{2}, \tau\right)} d B_{\tau}^{2},
\end{aligned}
$$

with initial condition $\{s, y\}$ at time $t$. In addition, $d B_{\tau}^{1} d B_{\tau}^{2}=\rho\left(\hat{\xi}_{\tau}^{1}, \hat{\xi}_{\tau}^{2}, \tau\right)$.

Suppose $s^{\prime}>s^{\prime \prime}$. Again $\hat{\xi}_{T}^{1 s^{\prime}, y, t} \geq \hat{\xi}_{T}^{1 s^{\prime \prime}, y, t}$ with probability 1 . If for all $s, g_{11} \geq 0$, then $g_{1}$ is non-decreasing in $s$. Hence $g_{1}\left(\hat{\xi}_{T}^{1 s^{\prime}, y, t}\right) \geq g\left(\hat{\xi}_{T}^{1 s^{\prime \prime}, y, t}\right)$ with probability one and

$$
v_{1}\left(s^{\prime}, y, t\right)=E\left\{g_{1}\left(\hat{\xi}_{T}^{1 s^{\prime}, y, t}\right)\right\} \geq E\left\{g_{1}\left(\hat{\xi}_{T}^{1 s^{\prime \prime}, y, t}\right)\right\}=v_{1}\left(s^{\prime \prime}, y, t\right) .
$$

Similarly, if for all $s, g_{11} \leq 0$, then $g_{1}$ is non-increasing in $s$. Hence $v_{1}\left(s^{\prime}, y, t\right) \leq v_{1}\left(s^{\prime \prime}, y, t\right)$.

\section{Proof of Theorem 3}

Combining $(A 6)$ and $(A 7)$ the quantity $\left[v(s, y, t)-v_{1}(s, y, t) s\right]$ can be shown to satisfy a p.d.e. of the form in $(A 2)$ subject to the terminal condition

$$
v(s, y, T)-v_{1}(s, y, T) s=g(s)-g_{1}(s) s .
$$

If $y$ is the price of a traded asset, the quantity $\left[v(s, y, t)-v_{1}(s, y, t) s\right]$ can be interpreted as the value of the non-stock position in a replicating portfolio. Under the conditions of Theorem 3 the terms $R(x, t)$ and $h(x, t)$ of $(A 2)$ simplify to $R(x, t)=r(t)$ and $h(x, t)=0$. Thus

$$
v(s, y, t)-v_{1}(s, y, t) s=e^{-\int_{t}^{T} r(\tau) d \tau} E\left\{g\left(\xi_{T}^{1 s, y, t}\right)-g_{1}\left(\xi_{T}^{1^{s, y, t}}\right) \xi_{T}^{1 s, y, t}\right\},
$$

where $\xi_{T}^{1}$ and $\xi_{T}^{2}$ solve a system of correlated SDE's with initial conditions $\{s, y\}$ at time t.

\section{Proof of Theorem 4}

The proof will be made more transparent by measuring the prices of time $T$ maturity contingent claims and of the underlying asset relative to the price of a pure discount bond maturing at time $T$; i.e., using the bond as numeraire. Using upper (lower) case notation to denote relative (absolute) price levels, the normalized prices take the form:

$$
\begin{aligned}
S_{t} & =e^{\int_{t}^{T} r(\tau) d \tau} s_{t}, \\
C\left(S_{t}, t\right) & =e^{\int_{t}^{T} r(\tau) d \tau} c\left(s_{t}, t\right)=e^{\int_{t}^{T} r(\tau) d \tau} c\left(e^{\int_{t}^{T}-r(\tau) d \tau} S_{t}, t\right) .
\end{aligned}
$$


The normalized call price has the following partial derivatives:

$$
\begin{aligned}
C_{1}(S, t) & =c_{1}(s, t) . \\
C_{11}(S, t) & =c_{11}(s, t) e^{-\int_{t}^{T} r(\tau) d \tau} .
\end{aligned}
$$

Note that convexity of $C(S, t)$ in $S$ for all $S$ and $t$ implies the convexity of $c(s, t)$ in $s$ for all $s$ and $t$, and vice-versa. Using the normalized pricing system, the option's value satisfies the following p.d.e.,

$$
C_{2}(S, t)+\frac{1}{2}[\Sigma(S, t) S]^{2} C_{11}(S, t)=0
$$

$$
\text { where } \Sigma(S, t):=\sigma\left(e^{-\int_{t}^{T} r(\tau) d \tau} S, t\right)=\sigma(s, t) \text {. }
$$

The transformation from $\sigma(\cdot)$ to $\Sigma(\cdot)$ is non-trivial. Other than in a deterministic volatility (BlackScholes) world, the transformation requires knowledge of $r(\tau)$ for all $\tau \in[t, T]$.

It is tempting to view $(A 10)$ as implying that one can determine the forward price of a contingent claim provided one knows the forward price of the underlying asset and $t$ without having to know anything about interest rates. But when the volatility of returns on the underlying asset depends on the spot price of the asset, then the $\Sigma(S, t)$ function masks, but does not remove, the relation between interest rates and the claim's forward price. ${ }^{A 1}$ Consider the following transformed price systems:

$$
\begin{aligned}
S_{t} & =s_{t} e^{\int_{t}^{T} r^{\mathcal{A}}(\tau) d \tau}, \\
C^{\mathcal{A}}\left(S_{t}, t\right) & =c^{\mathcal{A}}\left(S_{t} e^{-\int_{t}^{T} r^{\mathcal{A}}(\tau) d \tau}, t\right) e^{\int_{t}^{T} r^{\mathcal{A}}(\tau) d \tau}, \\
C^{\mathcal{B}}\left(S_{t}, t\right) & =c^{\mathcal{B}}\left(S_{t} e^{-\int_{t}^{T} r^{\mathcal{A}}(\tau) d \tau}, t\right) e^{\int_{t}^{T} r^{\mathcal{A}}(\tau) d \tau} .
\end{aligned}
$$

One can think of the transformation as setting the interest rate to zero in economy $\mathcal{A}$ and to $\Delta(\tau)=r^{\mathcal{B}}(\tau)-r^{\mathcal{A}}(\tau)$ in economy $\mathcal{B} . C^{\mathcal{A}}(S, t)$ solves the p.d.e.

$$
C_{2}^{\mathcal{A}}(S, t)+\frac{1}{2}[\Sigma(S, t) S]^{2} C_{11}^{\mathcal{A}}(S, t)=0
$$

$A 1$ If the volatility of percent changes in the forward price for delivery of the underlying asset at time $T$ depends on the level of the forward price for delivery at time $T$ (and not on the spot price), then the forward price of a call option with a time $T$ maturity will not depend on the term structure of interest rates. However, the forward prices of the set of call options maturing at dates other than $T$ will depend on the term structure of interest rates. 
subject to $C^{\mathcal{A}}(S, T)=\max [0, S-K]$, where $\Sigma(S, t)=\sigma\left(e^{-\int_{t}^{T} r^{\mathcal{A}}(\tau) d \tau} S, t\right)=\sigma(s, t) . C^{\mathcal{B}}(S, t)$ solves the p.d.e.

$$
C_{2}^{\mathcal{B}}(S, t)+\frac{1}{2}[\Sigma(S, t) S]^{2} C_{11}^{\mathcal{B}}(S, t)=-\Delta(t)\left(C_{1}^{\mathcal{B}}(S, t) S-C^{\mathcal{B}}(S, t)\right)
$$

subject to $C^{\mathcal{B}}(S, T)=\max [0, S-K]$. Let $X\left(S_{t}, t\right):=C^{\mathcal{B}}\left(S_{t}, t\right)-C^{\mathcal{A}}\left(S_{t}, t\right)$ denote the difference between the transformed values of the calls across the two economies. Note that $X\left(S_{t}, t\right)>0$ implies $c^{\mathcal{B}}\left(s_{t}, t\right)>c^{\mathcal{A}}\left(s_{t}, t\right)$.

From $(A 11)$ and $(A 12)$ we have

$$
X_{2}(S, t)+\frac{1}{2}[\Sigma(S, t) S]^{2} X_{11}(S, t)+\Delta(t)\left(C_{1}^{\mathcal{B}}(S, t) S-C^{\mathcal{B}}(S, t)\right)=0
$$

For a given value of $S_{T}$ at maturity, the time $T$ prices of the two calls coincide:

$$
X\left(S_{T}, T\right)=C^{\mathcal{B}}\left(S_{T}, T\right)-C^{\mathcal{A}}\left(S_{T}, T\right)=\max \left[0, S_{T}-K\right]-\max \left[0, S_{T}-K\right]=0
$$

Thus $X(S, t)$ is given by the solution to the p.d.e. in $(A 13)$ subject to the terminal condition $X(S, T)=0$. From the Feynman-Kac Theorem we have

$$
X(S, t)=E\left\{\int_{t}^{T} \Delta(\tau)\left(C_{1}^{\mathcal{B}}\left(\xi_{\tau}^{S, t}, \tau\right) \xi_{\tau}^{S, t}-C^{\mathcal{B}}\left(\xi_{\tau}^{S, t}, \tau\right)\right) d \tau\right\}
$$

where $\xi_{\tau}^{S, t}$ solves the SDE: $d \xi_{\tau}=v\left(\xi_{\tau}, \tau\right) \xi_{\tau} d B_{\tau}$, with initial condition $S$ at time $t$.

For $c^{\mathcal{B}}\left(s_{t}, t\right)=s_{t}$, the weak inequality is satisfied immediately since $c^{\mathcal{A}}\left(s_{t}, t\right) \leq s_{t}$. For $c^{\mathcal{B}}\left(s_{t}, t\right)=0$, it follows that for all $\tau \in[t, T], C^{\mathcal{B}}\left(\xi_{\tau}, \tau\right)=0$, and the integrand, $\Delta(\tau)\left(C_{1}^{\mathcal{B}}\left(\xi_{\tau}, \tau\right) S_{\tau}-\right.$ $\left.C^{\mathcal{B}}\left(\xi_{\tau}, \tau\right)\right)$, is zero for all $\tau \in[t, T]$. The weak inequality is then satisfied. Finally for $0<$ $c^{\mathcal{B}}\left(s_{t}, t\right)<s_{t}$, it follows from Theorem 2 that for all $\tau \in[t, T]$ and all $\xi_{\tau}>0,0 \leq C^{\mathcal{B}}\left(\xi_{\tau}, \tau\right)<\xi_{\tau}$. For $C^{\mathcal{B}}\left(\xi_{\tau}, \tau\right)=0$ the integrand is zero. For $0<C^{\mathcal{B}}\left(\xi_{\tau}, \tau\right)<\xi_{\tau}$, Proposition 1 implies that $C_{1}^{\mathcal{B}}\left(\xi_{\tau}, \tau\right) \xi_{\tau}-C^{\mathcal{B}}\left(\xi_{\tau}, \tau\right)>0$, and hence $\Delta(\tau)\left(C_{1}^{\mathcal{B}}\left(\xi_{\tau}, \tau\right) \xi_{\tau}-C^{\mathcal{B}}\left(\xi_{\tau}, \tau\right)\right)$ is non-negative, and strictly positive for $\Delta(\tau)>0$. Thus for $0<c^{\mathcal{B}}\left(s_{t}, t\right)<s_{t}$, since $\int_{t}^{T} \Delta(\tau) d \tau>0$,

$$
X(S, t)=E\left\{\int_{t}^{T} \Delta(\tau)\left(C_{1}^{\mathcal{B}}\left(\xi_{\tau}^{S, t}, \tau\right) \xi_{\tau}^{S, t}-C^{\mathcal{B}}\left(\xi_{\tau}^{S, t}, \tau\right)\right) d \tau\right\}>0
$$


Rather than apply the Feynman-Kac Theorem, the task of demonstrating that $X(S, t)>0$ can be transformed into a familiar, and intuitively positive, valuation problem. Suppose first that in the normalized (zero interest rate) economy $\mathcal{A}$, we wish to value a contingent claim, $V\left(S_{t}, t\right)$, with the following contractual terms: The party long the contract will at all times $\tau \in[t, T]$ receive a continuous income stream from the short equal to $\Delta(\tau)\left(C_{1}^{\mathcal{B}}\left(S_{\tau}, \tau\right) S_{\tau}-C^{\mathcal{B}}\left(S_{\tau}, \tau\right)\right)$, and nothing thereafter. Given the assumptions of Theorem 4 such an income stream is always nonnegative. Further, when $0<c^{\mathcal{B}}(s, t)<s$, the strict convexity result of Proposition 1 implies that the income stream will, with positive probability, be strictly positive over some time interval. Thus at time $t$ this income stream contract has a strictly positive value to the long; i.e., $V\left(S_{t}, t\right)>0$. At its maturity, the income stream contract is valueless, and $V\left(S_{T}, T\right)=0$. The p.d.e. and terminal condition for this income stream contingent claim are identical to the p.d.e. in $(A 13)$ and the terminal condition whose solution determines $X(S, t)$. It follows immediately that $X(S, t)=$ $V(S, t) \geq 0$. Further, provided $0<c^{\mathcal{B}}(s, t)<s, X(S, t)>0$.

\section{Proof of Theorem 5}

Suppose that changes in the value of a third underlying asset, $\mathcal{U}$, are also described by a one-dimensional diffusion, with $\sigma^{\mathcal{U}}(s, t)=\sigma^{\mathcal{A}}(s, t)=\sigma^{\mathcal{B}}(s, t)=\sigma(s, t)$. Suppose further that asset $\mathcal{U}$ pays a continuous proportional dividend at the rate $\delta^{\mathcal{U}}(\tau)$ for all $\tau \in[t, T]$. The superscript ' $\mathcal{U}$ ' is a mnemonic for underlying. Consider a call option written on asset $\mathcal{U}, c^{\mathcal{U}}(s, t)$, with the usual payoff at maturity of $\max \left[0, s_{T}^{\mathcal{U}}-K\right]$, and the additional contractual feature that at all times $\tau \in[t, T]$, the short pays the long a continuous dividend, proportional to the value of the call, at the rate $\delta^{\mathcal{O}}(\tau)$. The superscript ' $\mathcal{O}$ ' is a mnemonic for option. We introduce the following notation to describe this call: $c\left(s, t, \delta^{\mathcal{U}}, \delta^{\mathcal{O}}\right)$. Using this notation we have $c^{\mathcal{U}}(s, t)=c\left(s, t, \delta^{\mathcal{U}}, \delta^{\mathcal{O}}\right)$, $c^{\mathcal{A}}(s, t)=c\left(s, t, \delta^{\mathcal{A}}, 0\right)$, and $c^{\mathcal{B}}(s, t)=c(s, t, 0,0)$. To preclude arbitrage it must be that

$$
c^{\mathcal{U}}(s, t)=e^{\int_{t}^{T} \delta^{\mathcal{O}}(\tau) d \tau} c\left(s, t, \delta^{\mathcal{U}}, 0\right)
$$

The value $c^{\mathcal{U}}(s, t)$ is given by the solution of the following p.d.e.

$$
c_{2}^{\mathcal{U}}(s, t)+\frac{1}{2}[\sigma(s, t) s]^{2} c_{11}^{\mathcal{U}}(s, t)+r(t) c^{\mathcal{U}}(s, t)\left(\Omega^{\mathcal{U}}(s, t)-1\right)+\delta^{\mathcal{O}}(t) c^{\mathcal{U}}(s, t)-c_{1}^{\mathcal{U}}(s, t) \delta^{\mathcal{U}}(t) s=0,
$$


subject to the terminal condition $c^{\mathcal{U}}(s, T)=\max [0, s-K]$. Now suppose further that for all $\tau$, $\delta^{\mathcal{U}}(\tau)=\delta^{\mathcal{O}}(\tau)=\delta^{\mathcal{A}}(\tau)$. Substituting into the p.d.e. in $(A 15)$ gives

$$
\begin{gathered}
c_{2}^{\mathcal{U}}(s, t)+\frac{1}{2}[\sigma(s, t) s]^{2} c_{11}^{\mathcal{H}}(s, t)+r(t) c^{\mathcal{U}}(s, t)\left(\Omega^{\mathcal{U}}(s, t)-1\right)+\delta^{\mathcal{A}}(t) c^{\mathcal{U}}(s, t)-c_{1}^{\mathcal{U}}(s, t) \delta^{\mathcal{A}}(t) s \\
=c_{2}^{\mathcal{U}}(s, t)+\frac{1}{2}[\sigma(s, t) s]^{2} c_{11}^{\mathcal{U}}(s, t)+\left(r(t)-\delta^{\mathcal{A}}(t)\right) c^{\mathcal{U}}(s, t)\left(\Omega^{\mathcal{U}}(s, t)-1\right)=0 .
\end{gathered}
$$

Thus, the p.d.e. in (A16), whose solution determines the value of $c^{\mathcal{U}}(s, t)$, is identical to the p.d.e. that would determine the value of $c^{\mathcal{B}}(s, t)$ in an otherwise equivalent economy in which, at all times $\tau \in[t, T]$, the interest rate was lower than $r(\tau)$ by the amount $\delta^{A}(\tau)$. It then follows from Theorem 4 that, for all $s$ and $t$,

$$
c^{\mathcal{B}}(s, t) \geq c^{\mathcal{U}}(s, t)
$$

and, for all $s$ and $t$ such that $0<c^{\mathcal{B}}(s, t)<s$,

$$
c^{\mathcal{B}}(s, t)>c^{\mathcal{U}}(s, t) .
$$

Further, substituting $\delta^{\mathcal{U}}(\tau)=\delta^{\mathcal{O}}(\tau)=\delta^{\mathcal{A}}(\tau)$ into expression $(A 14)$ gives

$$
c^{\mathcal{U}}(s, t)=e^{\int_{t}^{T} \delta^{\mathcal{A}}(\tau) d \tau} c\left(s, t, \delta^{\mathcal{A}}, 0\right)=e^{\int_{t}^{T} \delta^{\mathcal{A}}(\tau) d \tau} c^{\mathcal{A}}(s, t) .
$$

Combining ( $A 17$ ) and (A18) gives, for all $s$ and $t$,

$$
c^{\mathcal{A}}(s, t) \leq e^{-\int_{t}^{T} \delta^{\mathcal{A}}(\tau) d \tau} c^{\mathcal{B}}(s, t)
$$

and, for all $s$ and $t$ such that $0<c^{\mathcal{B}}(s, t)<s$,

$$
c^{\mathcal{A}}(s, t)<e^{-\int_{t}^{T} \delta^{\mathcal{A}}(\tau) d \tau} c^{\mathcal{B}}(s, t) .
$$

\section{Proof of Theorem 6}

Let $S_{t}^{\mathcal{A}}$ and $S_{t}^{\mathcal{B}}$ denote the time $t$ normalized prices of the two assets.

$$
\begin{aligned}
& d S_{\tau}^{\mathcal{A}}=\alpha^{\mathcal{A}}(\cdot) d \tau+\Sigma^{\mathcal{A}}\left(S_{\tau}^{\mathcal{A}}, \tau\right) S_{\tau}^{\mathcal{A}} d B_{\tau} . \\
& d S_{\tau}^{\mathcal{B}}=\alpha^{\mathcal{B}}(\cdot) d \tau+\Sigma^{\mathcal{B}}\left(S_{\tau}^{\mathcal{B}}, \tau\right) S_{\tau}^{\mathcal{B}} d B_{\tau} .
\end{aligned}
$$


For all $S$ and $\tau, \Sigma^{\mathcal{B}}(S, \tau) \geq \Sigma^{\mathcal{A}}(S, \tau)$, and, for $S$ and $\tau$ in some region, $\Sigma^{\mathcal{B}}(S, \tau)>\Sigma^{\mathcal{A}}(S, \tau)$. Let $X(S, t):=V^{\mathcal{B}}(S, t)-V^{\mathcal{A}}(S, t)$ denote the difference in values of the normalized contingent claim prices. $V^{i}(S, t)$ solves

$$
V_{2}^{i}(S, t)+\frac{1}{2}\left[\Sigma^{i}(S, t) S\right]^{2} V_{11}^{i}(S, t)=0
$$

subject to $V^{i}(S, T)=g(S), i=\mathcal{A}, \mathcal{B} . X(S, t)$ solves

$$
X_{2}(S, t)+\frac{1}{2}\left[\Sigma^{\mathcal{A}}(S, t) S\right]^{2} X_{11}(S, t)+\frac{1}{2}\left[\left[\Sigma^{\mathcal{B}}(S, t)\right]^{2}-\left[\Sigma^{\mathcal{A}}(S, t)\right]^{2}\right] S^{2} V_{11}^{\mathcal{B}}(S, t)=0,
$$

subject to $X(S, T)=0$.

From Theorem 2 the term

$$
\frac{1}{2}\left[\left[\Sigma^{\mathcal{B}}(S, t)\right]^{2}-\left[\Sigma^{\mathcal{A}}(S, t)\right]^{2}\right] S^{2} V_{11}^{\mathcal{B}}(S, t)
$$

in the p.d.e. in (A19) is non-negative. The remainder of the proof parallels that of Theorem 4.

\section{Proof of the Strict Convexity Claim of Proposition 1}

Let $S$ and $C$ be the normalized prices of the underlying asset and the call as defined in the introductory paragraph of the Proof of Theorem 4. Suppose that at time $t^{\prime}$ the strict convexity claim is violated for prices in some region. Let $a\left(t^{\prime}\right)$ and $b\left(t^{\prime}\right) \geq a\left(t^{\prime}\right)$ denote the normalized prices marking the end points of that region; i.e., for all $S \in\left(a\left(t^{\prime}\right), b\left(t^{\prime}\right)\right], \max [0, S-K]<C\left(S, t^{\prime}\right)<S$ yet $C_{11}\left(S, t^{\prime}\right)=0$. Suppose $a\left(t^{\prime}\right)>0$. Since $C\left(a\left(t^{\prime}\right), t^{\prime}\right)>\max \left[0, a\left(t^{\prime}\right)-K\right]$, yet $C\left(a\left(t^{\prime}\right), T\right)=$ $\max \left[0, a\left(t^{\prime}\right)-K\right]$, there must exist a set of times $\tau \in\left(t^{\prime}, T\right]$ at which $C_{2}\left(a\left(t^{\prime}\right), \tau\right)<0$. Let us then consider the particular value of $t^{\prime}$ such that not only is $\max \left[0, a\left(t^{\prime}\right)-K\right]<C\left(a\left(t^{\prime}\right), t^{\prime}\right)<a\left(t^{\prime}\right)$ and $C_{11}\left(a\left(t^{\prime}\right), t^{\prime}\right)=0$, but for some $t^{\prime \prime}>t^{\prime}$ we have that for all $\tau \in\left(t^{\prime}, t^{\prime \prime}\right), C_{2}\left(a\left(t^{\prime}\right), \tau\right)<0$. Given the p.d.e. in $(A 10)$ we see immediately that for all $\tau \in\left(t^{\prime}, t^{\prime \prime}\right), C_{11}\left(a\left(t^{\prime}\right), \tau\right)>0$ and $\Sigma\left(a\left(t^{\prime}\right), \tau\right)>0$. Assume that $\Sigma\left(a\left(t^{\prime}\right), t^{\prime}\right)>0 .{ }^{A 2}$ Since $C_{2}\left(a\left(t^{\prime}\right), t^{\prime}\right)=0$ and $\Sigma\left(a\left(t^{\prime}\right), t^{\prime}\right)>0$, there then exists

A2 The condition $\Sigma\left(a\left(t^{\prime}\right), t^{\prime}\right)>0$ can be relaxed. The techniques used to analyze dynamic behavior near a local minimum are discussed in Wiener (1993). Observe that $[\Sigma(S, t) S]^{2}$ is a nonnegative function which can therefore have zero values only at local minimums. In any particular case, one should differentiate the p.d.e. in $(A 10)$ a sufficient number of times until the singularity is resolved. 
an arbitrarily small positive $\bar{\varepsilon}$ such that for all $\varepsilon \in(0, \bar{\varepsilon})$, not only is $C_{11}\left(a\left(t^{\prime}\right)-\varepsilon, t^{\prime}\right)>0$ and $\Sigma\left(a\left(t^{\prime}\right)-\varepsilon, t^{\prime}\right)>0$, but $C_{12}\left(a\left(t^{\prime}\right)-\varepsilon, t^{\prime}\right)>0$. Further, since $C_{2}\left(a\left(t^{\prime}\right), t^{\prime}\right)=0$ and for all $S, C_{2}\left(S, t^{\prime}\right) \leq 0$, it follows that $C_{12}\left(a\left(t^{\prime}\right), t^{\prime}\right)=0$. Strict convexity for all $\tau \in\left(t^{\prime}, t^{\prime \prime}\right)$ requires that for time $t^{\prime+}, \lim _{\varepsilon \rightarrow 0} \frac{C_{1}\left(a\left(t^{\prime}\right), t^{\prime+}\right)-C_{1}\left(a\left(t^{\prime}\right)-\varepsilon, t^{\prime}\right)}{\varepsilon}>0$. But since $C_{12}\left(a\left(t^{\prime}\right), t^{\prime}\right)=0$, $\lim _{\varepsilon \rightarrow 0} \frac{C_{1}\left(a\left(t^{\prime}\right), t^{\prime+}\right)-C_{1}\left(a\left(t^{\prime}\right)-\varepsilon, t^{\prime+}\right)}{\varepsilon}=\lim _{\varepsilon \rightarrow 0} \frac{C_{1}\left(a\left(t^{\prime}\right), t^{\prime}\right)-C_{1}\left(a\left(t^{\prime}\right)-\varepsilon, t^{\prime+}\right)}{\varepsilon}$, which, since $C_{12}\left(a\left(t^{\prime}\right)-\varepsilon, t^{\prime}\right)>$ 0 , is not greater than $\lim _{\varepsilon \rightarrow 0} \frac{C_{1}\left(a\left(t^{\prime}\right), t^{\prime}\right)-C_{1}\left(a\left(t^{\prime}\right)-\varepsilon, t^{\prime}\right)}{\varepsilon}=C_{11}\left(a\left(t^{\prime}\right), t^{\prime}\right)=0$, and we have a contradiction. Analogous arguments rule out a finite value for $b\left(t^{\prime}\right)$. Finally, the possibility that $a\left(t^{\prime}\right)=0$ and $b\left(t^{\prime}\right)$ is infinite is equivalent to the internally contradictory claim that for all $S>0$, $\max [0, S-K]<C\left(S, t^{\prime}\right)<S$, yet $C_{11}\left(S, t^{\prime}\right)=0$.

\section{References}

Arnold, Ludwig, 1992, Stochastic Differental Equations: Theory and Evidence (Krieger Publishing Company, Malabar, Fla.).

Avellaneda, Marco, Arnon Levy, and Antonio Parás, Pricing and hedging derivative securities in markets with uncertain volatilities, Undated Working Paper, Courant Institute of Mathematical Sciences.

Bick, Avi, 1982, Comments on the valuation of derivative assets, Journal of Financial Economics $10,331-345$.

Black, Fischer, and Myron Scholes, 1973, The pricing of options and corporate liabilities, Journal of Political Economy 81, 637-659.

Cox, John C., and Stephen A. Ross, 1976, A survey of some new results in financial option pricing theory, Journal of Finance 31, 383-402.

Cox, John C., and Mark Rubinstein, 1985, Options Markets (Prentice Hall, Inc., Englewood Cliffs, N.J.).

Dixit, Avinash K., and Robert S. Pindyck, 1994, Investment under Uncertainty (Princeton University Press, Princeton, N.J.).

El Karoui, Nicole, Monique Jeanblanc-Picque, and Ravi Visvanathan, On the robustness of BlackScholes equation, Undated Working Paper, Laboratoire de Probabilités, Université Pierre et Marie Curie.

El Karoui, Nicole, Monique Jeanblanc-Picqué, and Steven E. Shreve, 1995, Robustness of the Black and Scholes Formula, Working Paper, Laboratoire de Probabilités, Université Pierre et Marie.

Fischer, Edwin O., Robert Heinkel, and Josef Zechner, 1989, Dynamic capital structure choice: Theory and tests, Journal of Finance 44, 19-40.

Geske, Robert, 1979, The valuation of compound options, Journal of Financial Economics 7, 63-82. 
Grundy, Bruce D., 1991, Option prices and the underlying asset's return distribution, Journal of Finance 46, 1045-1069.

Jagannathan, Ravi K., 1984, Call options and the risk of underlying securities, Journal of Financial Economics 13, 425-434.

Karlin, Samuel, and Howard M. Taylor, 1981, A Second Course in Stochastic Processes (Academic Press, San Diego, Ca.).

Merton, Robert C., 1973, Theory of rational option pricing, Bell Journal of Economics and Management Science 4, 141-183.

Merton, Robert C., 1976, Option pricing when underlying stock returns are discontinuous, Journal of Financial Economics 3, 125-144.

Mohammed, S.E.A., 1978, Retarded Functional Differential Equations: A Global Point of View (Pitman Publishing, London, UK.).

Rothschild, Michael, and Joseph E. Stiglitz, 1970, Increasing risk I: A definition, Journal of Economic Theory 2, 225-243.

Rubinstein, Mark, 1983, Displaced diffusion option pricing, Journal of Finance 38, 213-217.

Wiener, Zvi, 1993, Instability with two zero frequencies, Journal of Differential Equations 103 , $58-68$. 


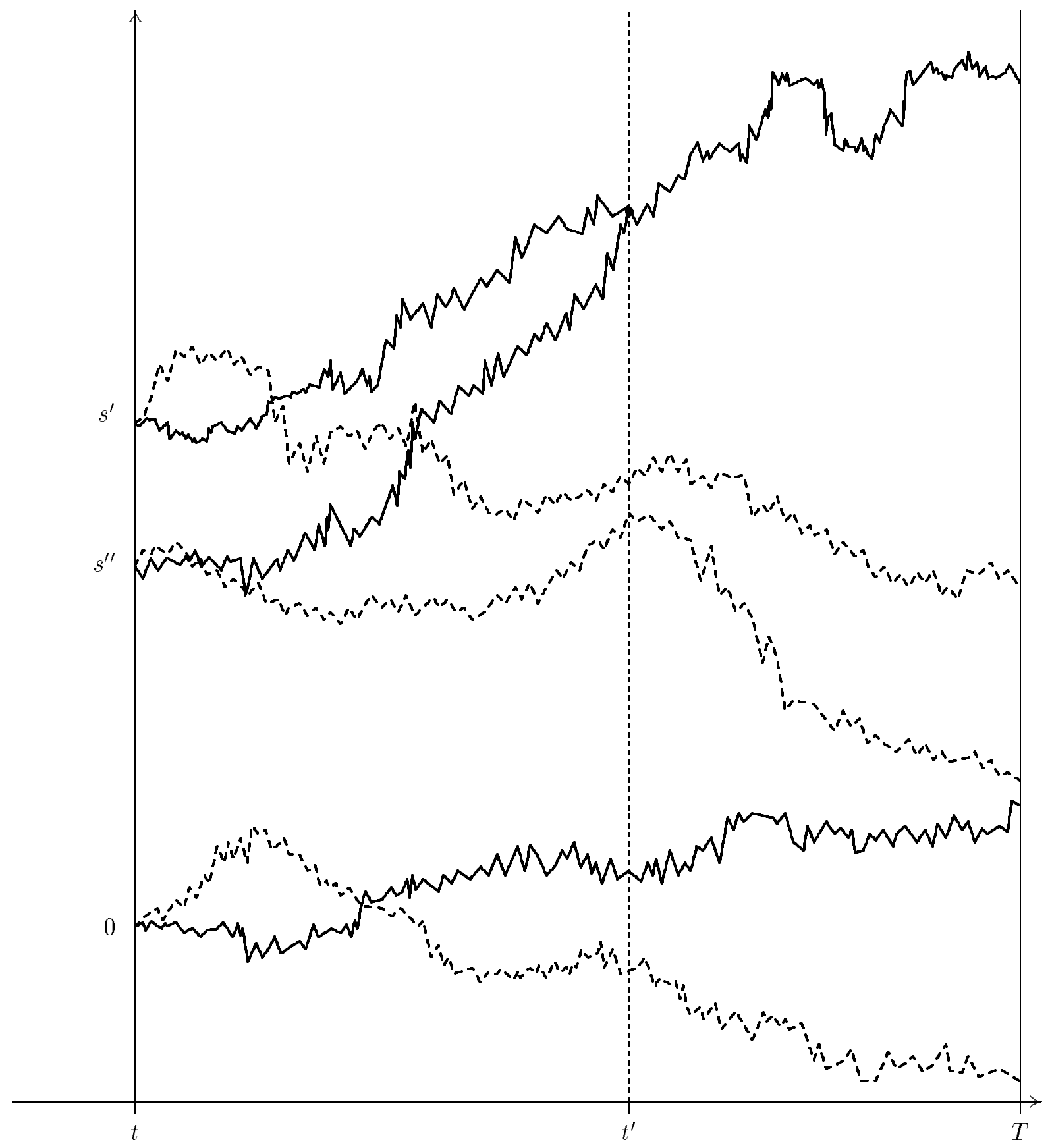

Figure 1. Illustration of the 'no-crossing' property. The lowest two paths are two sample paths of the driving Brownian motion. The solid risk-neutralized path drives the two solid sample paths of the risk-neutralized price process. The solid risk-neutralized path starting at $s^{\prime}$ stays above the path starting at $s^{\prime \prime}$ until, upon meeting, they fuse and merge onward. Similarly the dashed Brownian motion drives the two dashed sample paths of the risk-neutralized price process. They also exhibit the 'no-crossing' property. 


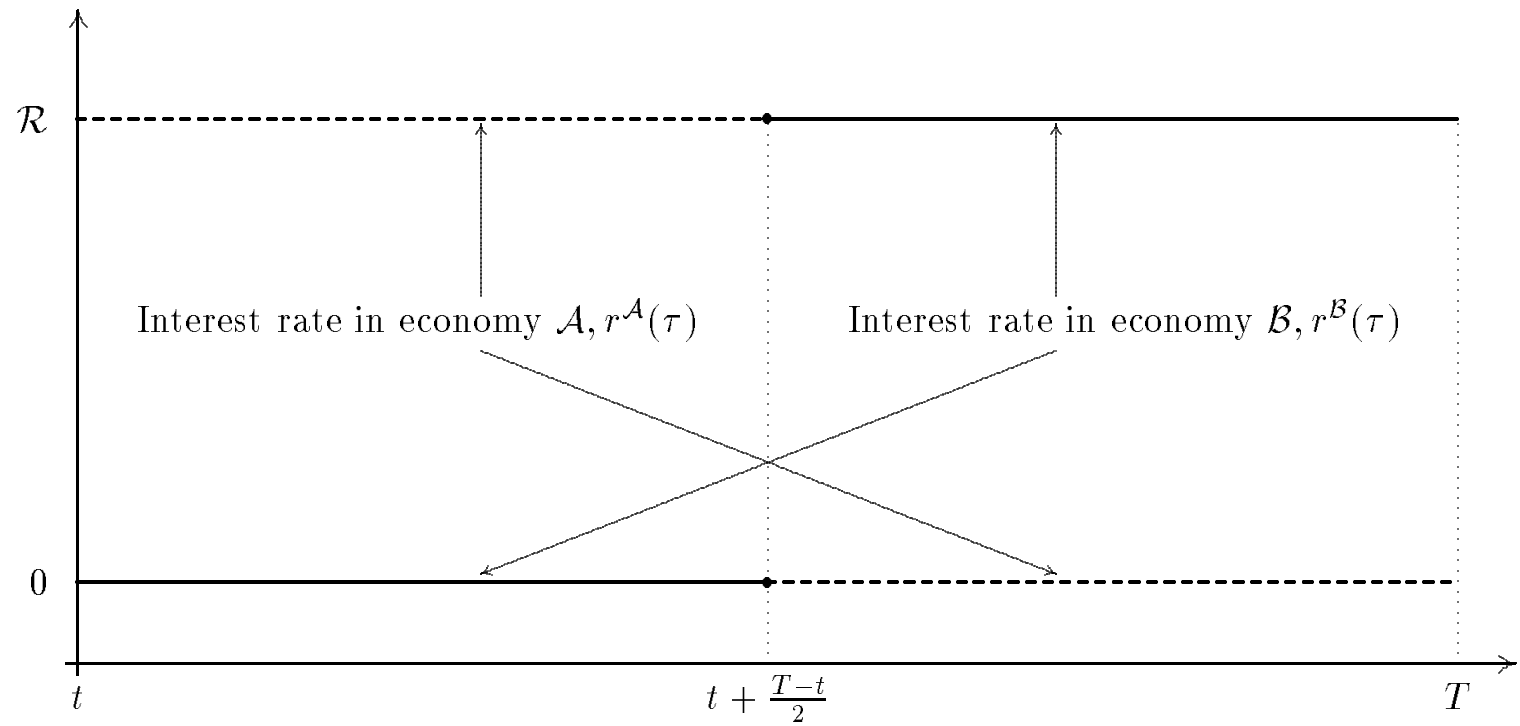

Figure 2a. Interest rate time profiles in two otherwise equivalent economies $\mathcal{A}$ and $\mathcal{B}$. One profile is a time permutation of the other with $\int_{t}^{T} r^{\mathcal{A}}(\tau) d \tau=\frac{T-t}{2} \mathcal{R}=\int_{t}^{T} r^{\mathcal{B}}(\tau) d \tau$. Therefore riskless bonds that promise the same payoff at time $T$ have the same price in both economies.

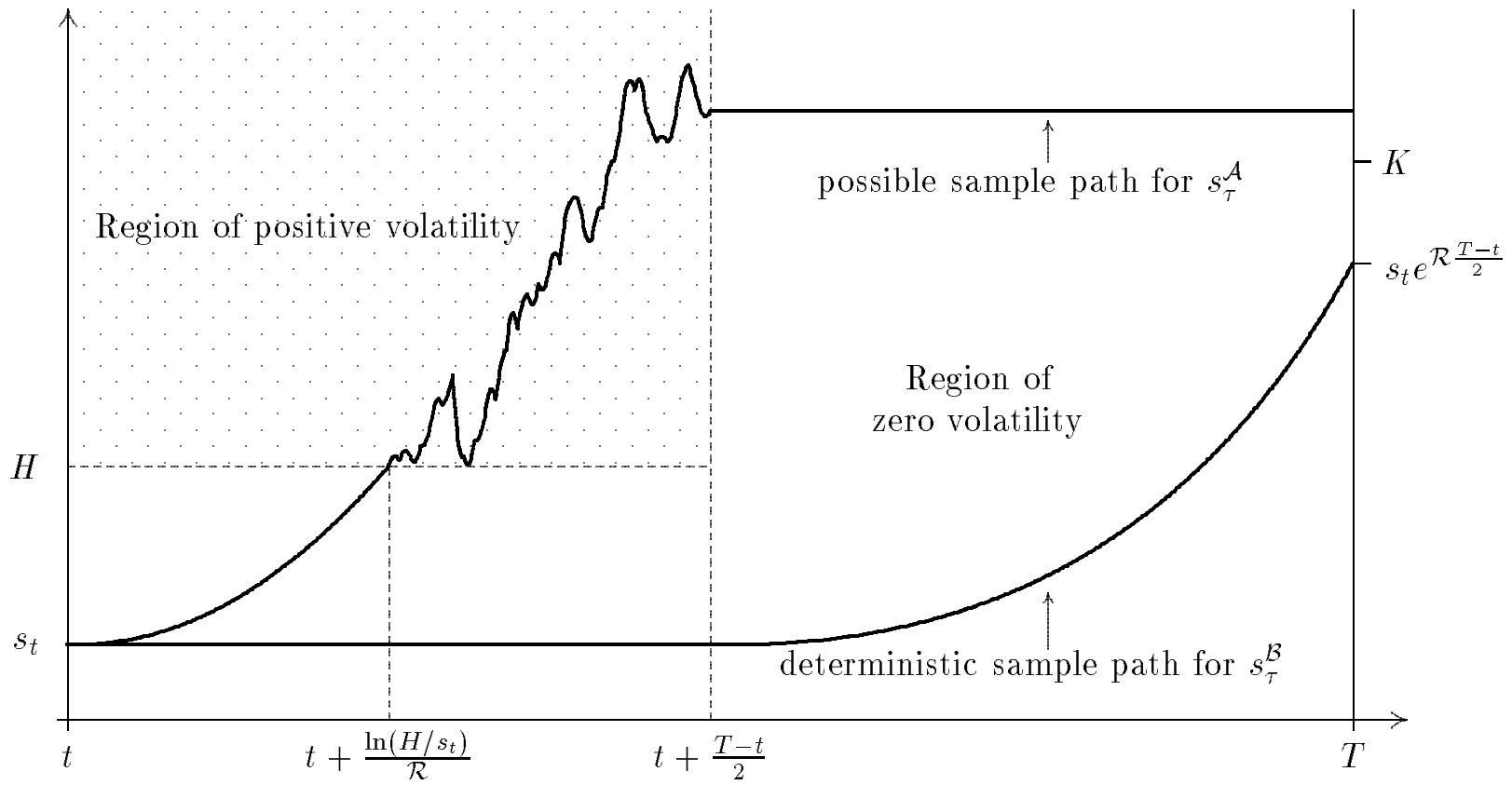

Figure $2 \mathrm{~b}$. Sample paths of an underlying asset's price process in economies $\mathcal{A}$ and $\mathcal{B}$. Both paths commence at the same level $s_{t}$ at time $t$, and both are driven by the same Brownian motion. In economy $\mathcal{A}$ the price ends above $K$ at time $T$. Due to the different interest rate time-profile in economy $\mathcal{B}$ and the functional form of the volatility, the price always finishes below $K$ in economy $\mathcal{B}$. 


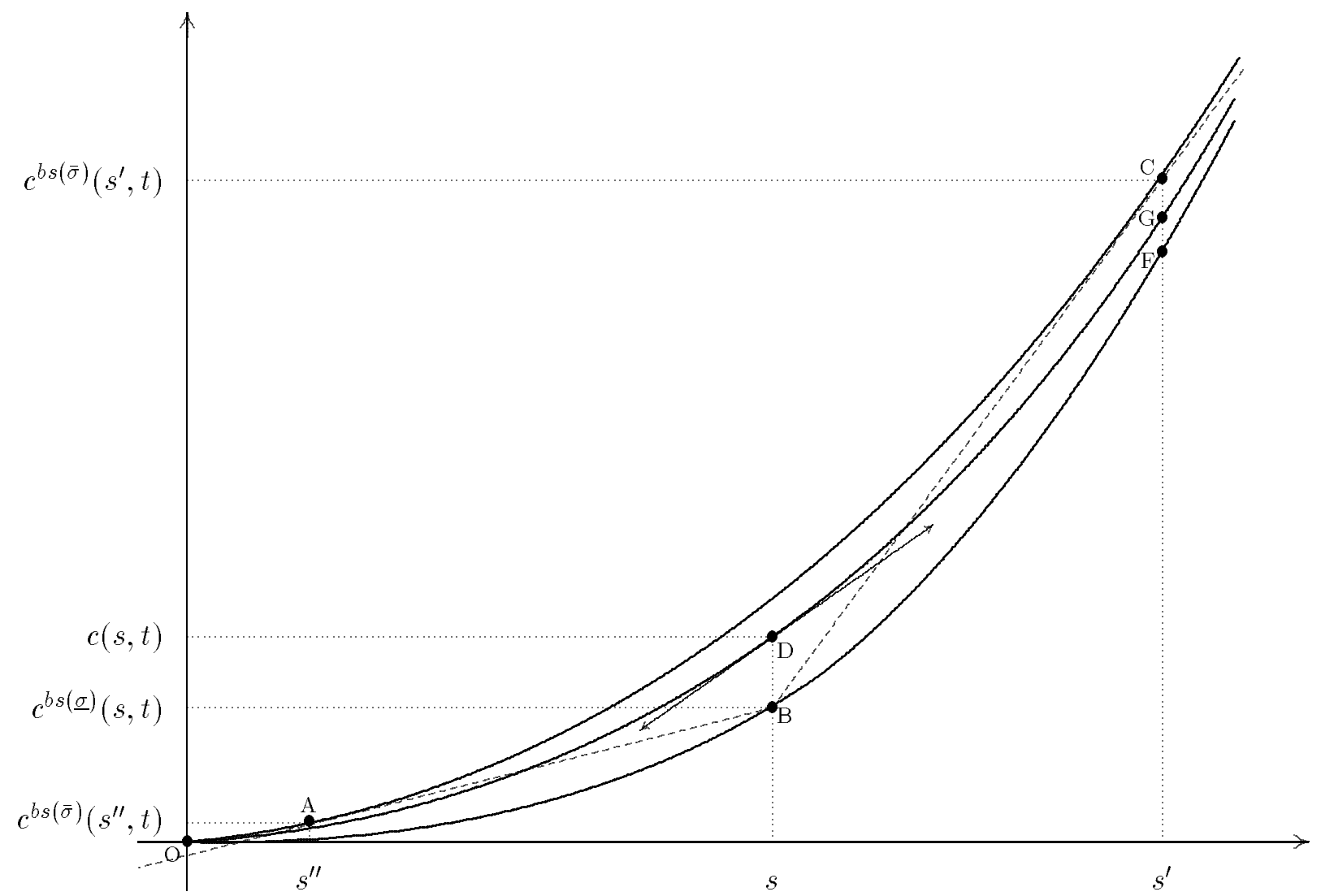

Figure 3. Bounds on a call's price and delta. The curve OAC (respectively, $\mathrm{OBF}$ ) is the Black-Scholes call price computed as a function of the underlying price using the upper bound, $\bar{\sigma}(t)$ (lower bound, $\underline{\sigma}(t)$ ), on the true volatility. The two curves bound the true call price curve ODG. The true hedge ratio given a stock price of $s$ is bounded from above by the slope of $\mathrm{BC}$ and from below by the slope of $\mathrm{AB}$; both tangents from $\mathrm{B}$ to OAC. $c(s, t)$ is the time $t$ value of a call option written on a asset worth $s \cdot c^{b s(\bar{\sigma})}(s, t)$ is the time $t$ Black-Scholes value of a call option written on an asset worth $s$ when the asset's volatility is the deterministic function $\bar{\sigma}(t) . c^{b s(\underline{\alpha})}(s, t)$ is similarly defined. 


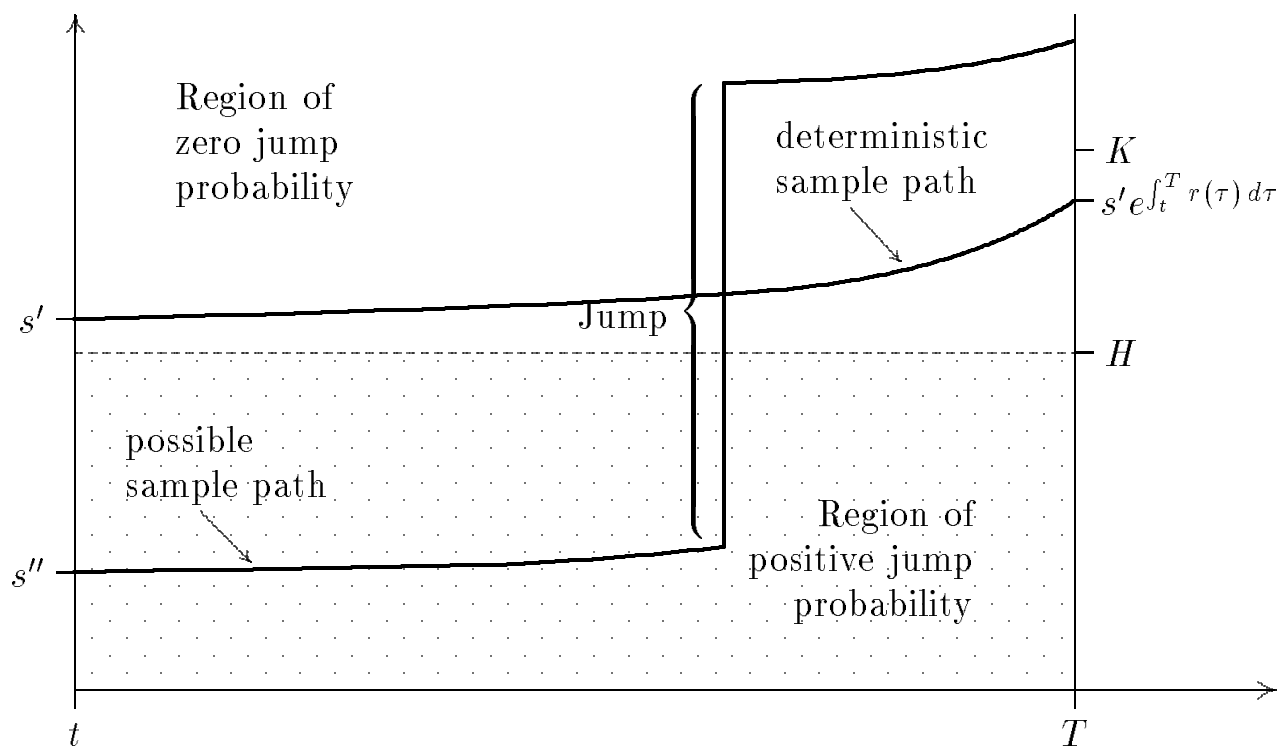

Figure 4a. Two sample paths of a non-proportional mixed diffusion-jump process for which the 'no-crossing' property is violated. The path that starts out lower has a positive probability of finishing above $K$ at time $T$. The path that starts out higher always finishes below $K$. A call option with exercise price $K$ and time $T$ maturity will be such that $c\left(s^{\prime}, t\right)=0$, yet $c\left(s^{\prime \prime}, t\right)>0$.

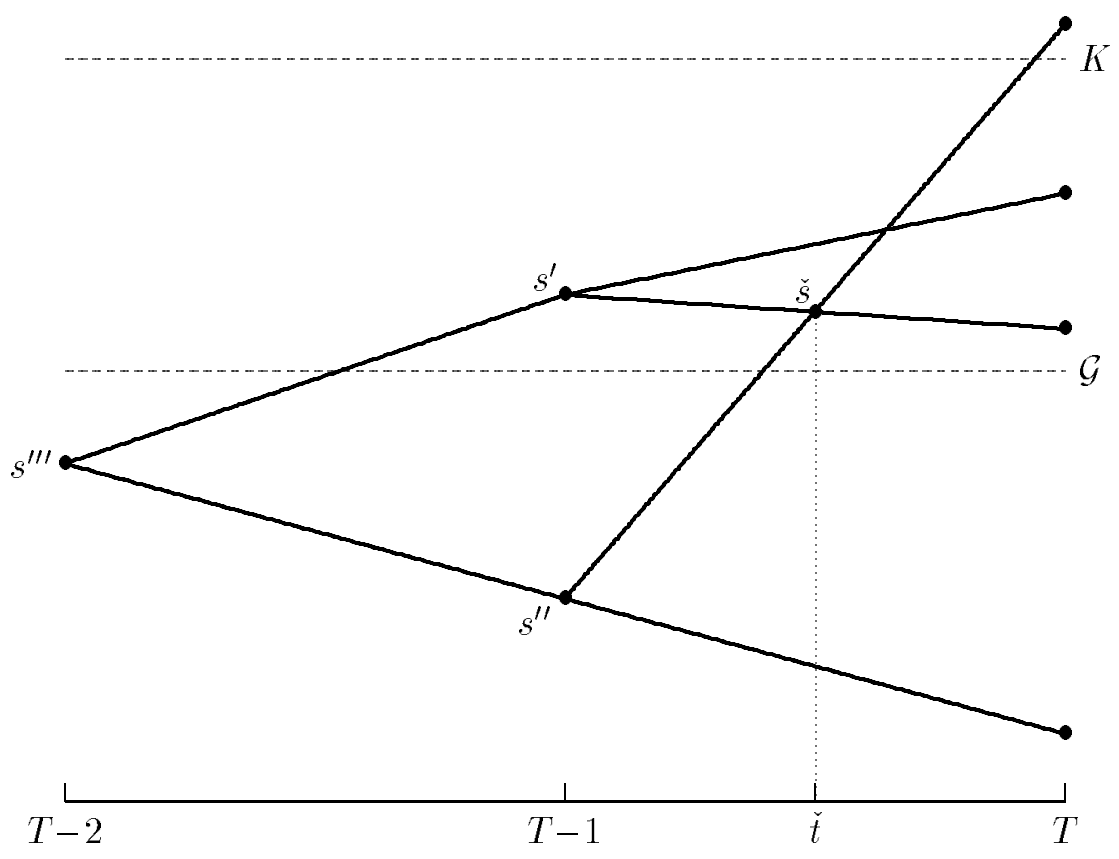

Figure 4b. A non-recombining binomial tree for which the 'no-crossing' property is violated. If the process reaches $s^{\prime}$, it will always finish below $K$ and a call with an exercise price of $K$ and a time $T$ maturity will be valueless. But if the process reaches $s^{\prime \prime}$, the process can finish above $K$ and the call has a positive price. 


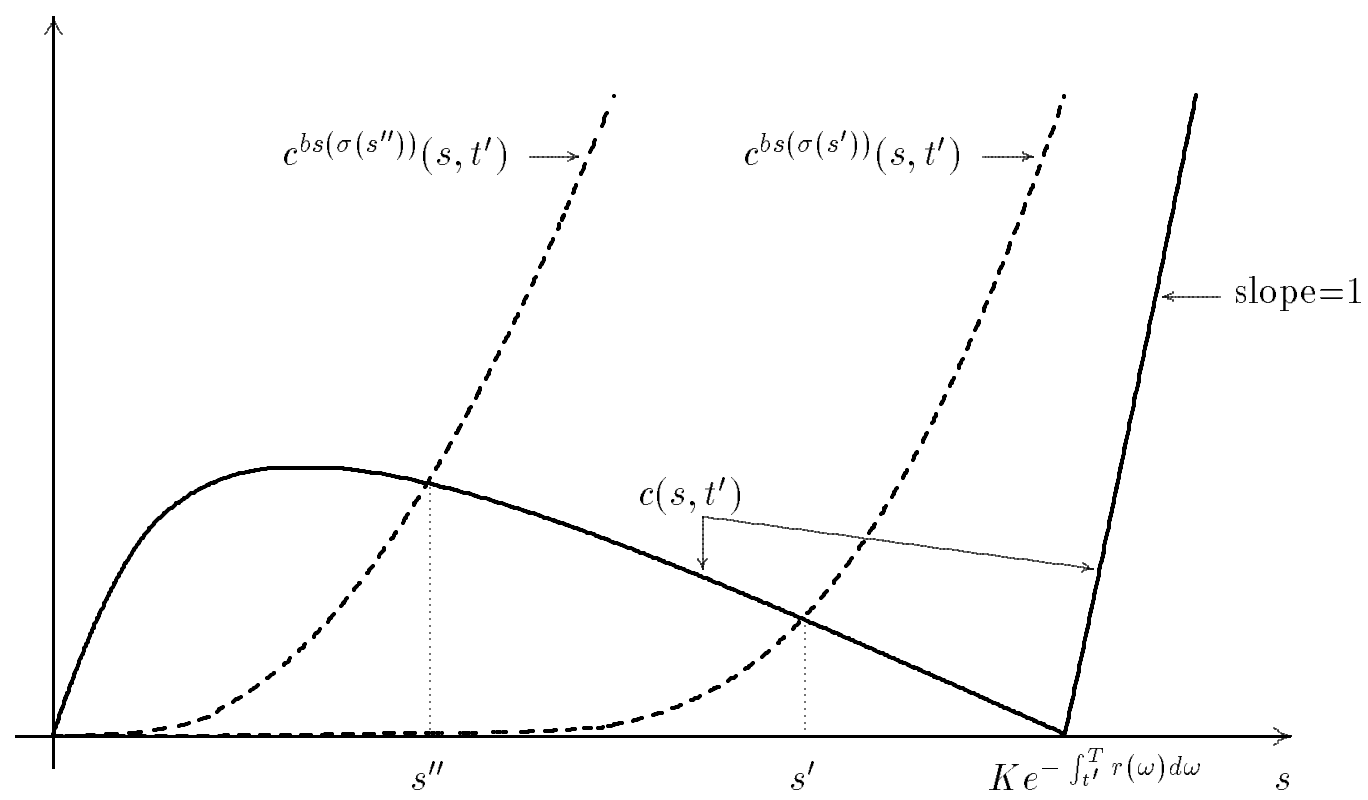

Figure 5a. The relation between $c\left(s, t^{\prime}\right)$ and $s$ in a non-Markovian setting. The call price function is non-monotonic and non-convex. $c\left(s, t^{\prime}\right)$ is the time $t^{\prime}$ value of a call option written on a asset worth $s . c^{b s\left(\sigma\left(s^{\prime}\right)\right)}\left(s, t^{\prime}\right)$ is the time $t^{\prime}$ Black-Scholes value of a call option written on an asset worth $s$ when the asset's volatility is constant and equal to $\sigma\left(s^{\prime}\right) . c^{b s\left(\sigma\left(s^{\prime \prime}\right)\right)}\left(s, t^{\prime}\right)$ is similarly defined.

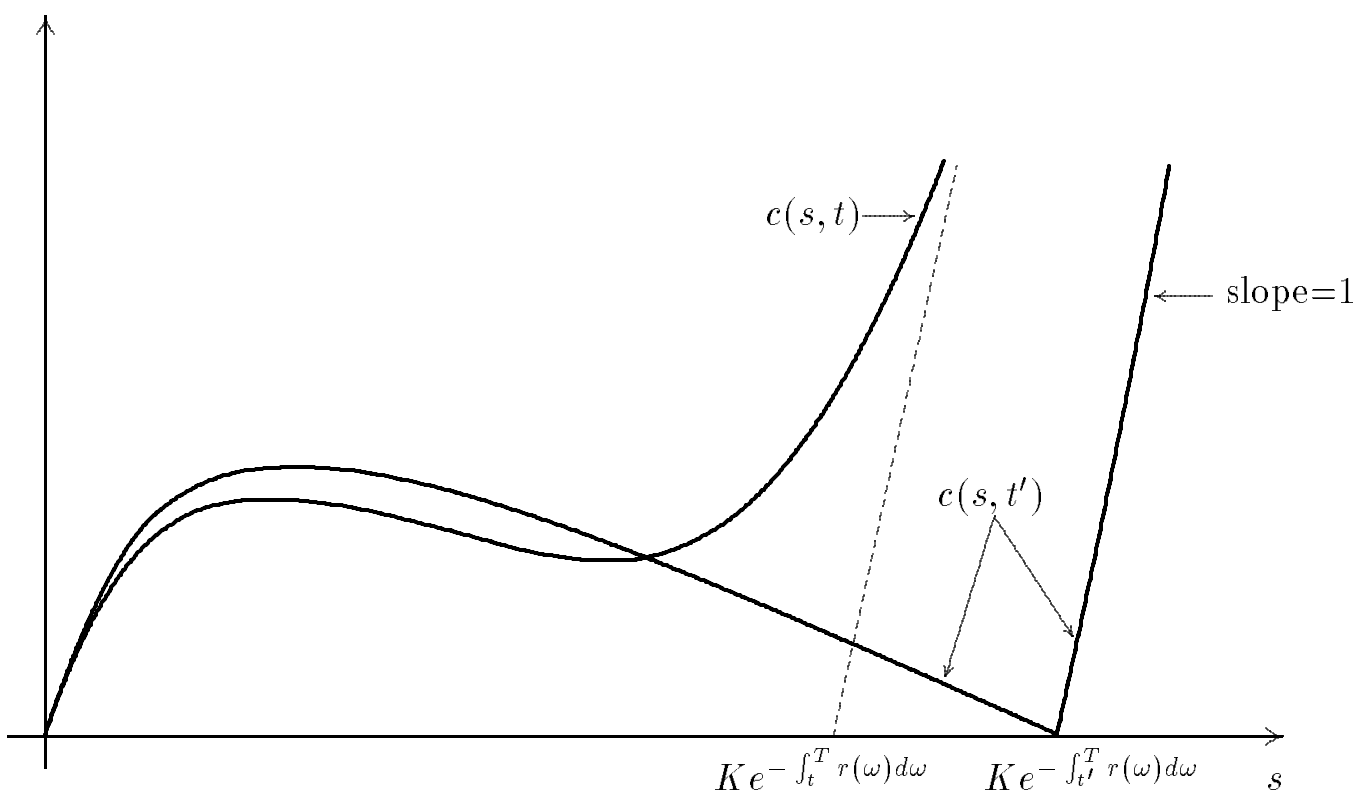

Figure $5 \mathbf{b}$. The relation between $c(s, \tau)$ and $\tau$ in a non-Markovian setting. In some region of the underlying asset price, the call option price is greater at a later time $t^{\prime}$ than at an earlier time $t$. Unlike in the BlackScholes world, the call is not uniformly a "wasting" asset. 


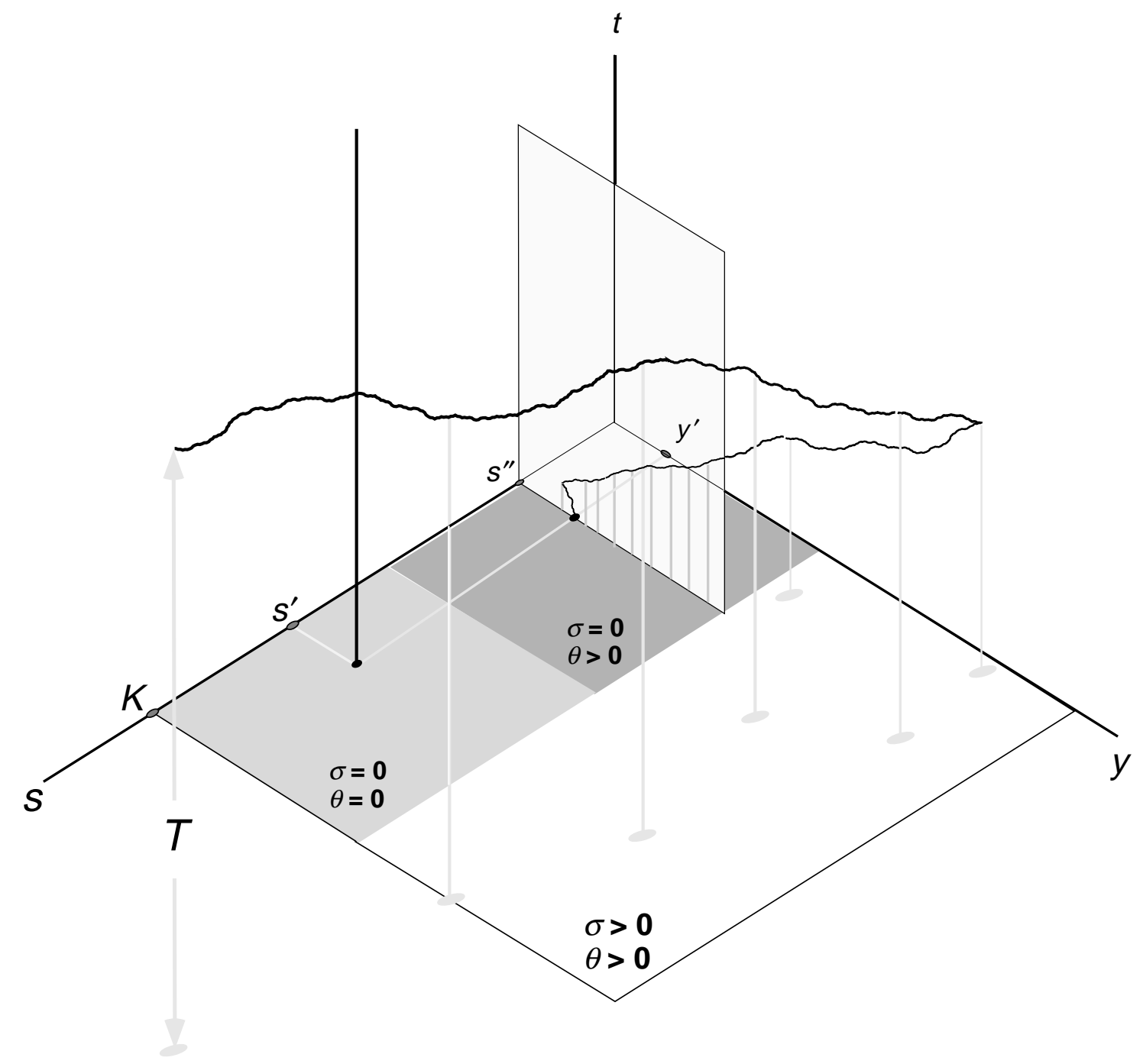

Figure 6. Two sample paths of a two-dimensional diffusion for which the 'no-crossing' property is violated. Shown are two sample paths of a zero-drift two-dimensional diffusion: a stock price process $s$, and the process $y$ which drives the stochastic volatility of $s$.

$$
\begin{aligned}
d s_{t} & =\sigma\left(s_{t}, y_{t}, t\right) s_{t} d B_{t}^{1} . \\
d y_{t} & =\theta\left(s_{t}, y_{t}, t\right) d B_{t}^{2} .
\end{aligned}
$$

At time 0 , one path starts at $\left(s^{\prime}, y^{\prime}\right)$ in the (light grey) region where everything is deterministic. It must then stay constant at its starting values, and the stock price component $s$ can not reach a value greater than $K$. The other sample path starts from $\left(s^{\prime \prime}, y^{\prime}\right)$ in the (dark grey) region where the $y$ component is stochastic. From these starting values there is a positive probability that the vector process will reach the (white) region where both $s$ and $y$ have positive volatities. From that region it is then possible for $s$ to reach a value above $K$. Therefore, for a call with an exercise price of $K, c\left(s^{\prime}, y^{\prime}, 0\right)=0$, yet $c\left(s^{\prime \prime}, y^{\prime}, 0\right)>0$. 